\title{
Head Nurses' Evaluation and Nurses' Self Evaluation for Performing Chest Nursing Care Procedures
}

\author{
Azza E. Elamia,Master degree in nursing service adminstration ,Faculty of nursing ,Tanta univeristy \\ Fouada M. Shabaan, Professor of Nursing Service Administration, Faculty of Nursing, Tanta University \\ Nagwa Ragab Atia, Prof. of Nursing Medical Surgical department, Faculty of Nursing, Tanta University \\ Maha E.Skokier, Lecturer of Nursing Services Administration, Faculty Nursing, Tanta University
}

\begin{abstract}
Background : Head nurse evaluation of the nurses' performance of chest care procedures is vital to quality patient care at ICU. While nurses' self-evaluation help nurses to pay attention to their own performance, raise their self-awareness and efficacy to make efforts for improvement in performing of six chest care procedures. So head nurses and nurses actually need an educational training program for enforcing them for continuous performance improvement and updating knowledge .Objective: study head nurses' evaluation and nurses' self -evaluation of performing chest nursing care procedures. Setting: Tanta University Hospitals at Mobark ,Chest , and Tanta University International Educational Hospital. Tools two tools used Tool I: Evaluation and self -evaluation for nurses' actual performance of chest nursing care procedures observation checklists. And tool II: Knowledge assessment of chest nursing care procedures steps, evaluation and self -evaluation process. Result Preprogram researcher head nurses evaluation and nurses self -evaluation showed that nurses performance were at unsatisfactory level for all items of the six chest care procedures. Head nurses $55 \%, 95 \%, 100 \%$ and $85 \%$ showed poor level for plan ,needed information ,principles of evaluation and methods ,respectively . All head nurses evaluation and nurses self- evaluation level of knowledge pre- program was poor . Actually no one of nurses showed excellent level preprogram for performing items of vibration, coughing and suctioning procedures which changed post program to $65 \%, 78.8 \%$ and $100 \%$, and at 3 month post program changed to $65 \%, 83.3 \%$ and $100 \%$ respectively. All head nurses and $80 \%$ of nurses had poor overall knowledge about six chest care procedure standard evaluation and self- evaluation principles preprogram .Implementation of educational program improved head nurses and nurses overall knowledge about chest care, evaluation and nurses self evaluation statistically significant at $\mathrm{P}<0.01$. Conclusion: Head nurses at Tanta university hospital were not evaluating nurses performance of chest care procedures leading to unsatisfactory nurses performance .Actually head nurses have insufficient knowledge to evaluate nurses performance and nurses have insufficient knowledge to perform chest care procedures according standard and cannot make self-evaluation for their performance. Implementation of educational program about evaluation principles,chest care procedure standard and selfevaluation principles successfully improved nurses staff performance and knowledge. Recommendation: Conduct regular training programs workshops and seminars for head nurses to update their knowledge and skills related to evaluation of nurses performance. Conduct periodical in service training program for nurses to refresh their knowledge and skills related to chest care procedure performance according to standard of its performance. Head nurses should encourage nurse self-evaluate their performance and provide supportive health work environment to help nurses improved their performance of chest care procedures .
\end{abstract}

Key words head nurse evaluation, nurses self- evaluation. Chest care procedures 


\section{Tanta Scientific Nursing Journal}

\section{Introduction}

Nursing is a professional issue and is central to patient care outcomes and which caring, the basis of good nursing. Presentday health services are highly complex and high quality care is mandatory. In order to face these challenges and ensure that the best care is given by nurses, it is necessary to evaluate the nurses' performance competence ${ }^{(1)}$. Nurses play a crucial role in patient-care and rapidly changing complex health care environment, there is a crucial demand for head nurses capable of making evaluation $^{(2)}$.

Head nurse performance is very significant because of their strong effect on the nurses. They interact with most nurses on an everyday basis and must action like role models, if their role weak unsuccessful to stimulate their nursing staff, it may affect dramatically the performance of the nurses ${ }^{(3)}$.Head nurse is labeled as a fulcrum of managerial success of the nursing unit and as having a pivotal role in the hospital as a whole. She has three main areas that constitute her role $^{(4)}$.

Make staff management to utilize, guide, evaluate and correct staff nurses in their nursing practice to ensure its smooth running to fulfill hospital goals for improving nursing staff performance .So increasing the quality of nursing care, head nurse are challenging to do efforts to get efficient and effective patient outcome ${ }^{(4)}$.Head nurses encourage nurses self-evaluation as the critical factor in improving performance standards within their hospital . It provides nurses with the capacity to predict and cope with change effectively. Head nurses good performance evaluation of nurses points out areas where nurses need to improve their self-performance ${ }^{(5)}$.

Evaluation in the healthcare context can be a complicated activity and some of the potential challenges of evaluation are described, alongside possible solutions. Further resources and guidance on evaluation activity to support nurses' ongoing development are identified ${ }^{(6)}$. Evaluation of chest care procedures delivery is an important aspect of nursing practice is being increasingly used and led by head nurses. It aims to encourage nurses think about how self- evaluation of practice activity of procedure performance and to consider why and how they should use self -evaluation in their practice ${ }^{(7)}$.

Frequently chest care techniques, such as turning, postural drainage percussion, vibration, coughing exercise and suctioning employed in a critical care 


\section{Tanta Scientific Nursing Journal}

units. Nurses are primarily responsible for their provision; also implemented these procedures and must consider chest care to be a part of their role, or how they perceive their knowledge and confidence pertaining to these techniques ${ }^{(8)}$. Intensive care practice is a very demanding multidisciplinary environment where nurses and head nurses are vital members. The early application of procedures can improve patient's thereby preventing some of the ICU-associated complications $^{(9)}$.

Self-evaluation is a systematic process involving the nurse and process of selfevaluation: is ongoing and sharplyfocused, involves monitoring and evaluating the effectiveness of existing provision and staff nurse achievements the need to have a clear and agreed view of the hospital development, to identify priorities which will have a positive effect on performance which requires the nurse to evaluate their performance critically ${ }^{(10)}$. A systematic process of self-evaluation as review, identify strengths and areas for improvement , prioritize areas for improvement, plan and implement action for improvement monitor and evaluate outcomes ${ }^{(11)}$.

Not having an effective evaluation system increases the risk of inefficiency, poor morale so nurses are critical to delivery of high-quality and efficient care relevant to nursing standard ${ }^{(12)}$. The nurses need to know exactly what is necessary to provide high-quality nursing care and that measures are in place to determine whether care meets the standards. Competence in nursing practice must be evaluated by the nurse's practices selfevaluation and head nurses supervisor ${ }^{(13)}$. So the aim of present study is to improve head nurses and nurses knowledge about chest care performance procedure standard, principles of evaluation and selfevaluation.

Aim of research to study head nurses evaluation and nurses' self- evaluation of performing chest nursing care procedures.

\section{Research hypothesis}

1-knowledge of head nurses and nurses about chest nursing care standard procedure will be changed.

2- Evaluation skill of head nurses will be enforced for evaluating nurses' performance for chest nursing care procedures.

3-Self-evaluation skill of nurses will be enforced for self-evaluating of their performance of chest nursing care procedures.

Design: Quasi-experimental research design was used to achieve the aim of 
research to fits the study nature about head nurses' evaluation and nurses' self evaluation of performing chest nursing care procedures at intensive care units.

Setting study was conducted at intensive care units (ICU) of Tanta University Hospitals at Mobark Hospital ,Chest Hospital and Tanta University International Educational Hospital. Mobark (ICU) capacity (16) beds, Chest Hospital (ICU) bed capacity ( 15 ) and Tanta University International Educational Hospital (ICU) bed capacity (15) bed .

Subjects All $(\mathrm{n}=20)$ head nurses and all $(\mathrm{n}=80)$ nurses working at previous mentioned (ICUs) at Tanta University Hospitals. The subject from Mobark hospital ICU $(n=6)$ head nurses and $(n=$ 27) nurses, from Chest Hospital ICU. ( $n=$ 8) head nurses and $(n=30)$ nurses and from Tanta University International Educational Hospital ICU. $(n=6)$ head nurses and $(n=23)$ nurses. Tools Data was collected using two tools to fulfill the purpose of study.

Tool I: Evaluation and self -evaluation for nurses' actual performance of chest nursing care procedures observation checklists. This tool developed by researcher guided by Langenderfer (1998) ${ }^{(14)}$, Gass (2009)
(15) and related recent literature. The tool used by researcher and head nurses to evaluate nurses' performance as well as used by nurses for self- evaluation. The tool used to assess the actual nurses' performance of chest nursing care 6 procedures (turning, postural drainage, chest percussion, vibration, cough techniques and suctioning) and composed of two parts as follows: Part one: Subject characteristic of nursing staff as hospital name, age, marital status, years of experience, level of education, attendance of educational program and positions.

Part two: Chest nursing care procedures :

Head nurses' evaluation observation, checklists. Procedure phases of the six chest care procedures namely turning, postural drainage, chest percussion, vibration, cough techniques and suctioning. Each procedure have specific checklist include items on the following: 1-Assessment indications and contraindications. 2-Preparation of equipment.3-Preparation of patient by communication and teaching.4Implementation of procedure and following infection control.5-Evaluation desirable and undesirable outcomes. This part was used to assess the nurses performance of chest nursing care 
procedure according to researcher and head nurses evaluation, it include six chest care procedures as follow: Turning patient includes 20 items (1-20).Postural drainage of patient includes 23 items (1-23). Percussion of patient includes 36 items (136).Vibrations of patient includes 19 items (1-9).Cough exercise of patient includes 19 items (1-19).Suctioning of patient includes 36 items (1-36).

Part three: Chest nursing care procedures: Nurses' self -evaluation.The same 6 check lists as parts two used for nurses self- evaluation of performing six chest care procedures.

Scoring system Part two and three the levels of head nurses and researcher evaluation and nurses self- evaluation of the six chest care procedures measured by three points Likert Scale (0-2), were complete done $=(2)$, partially done $=(1)$ and incorrectly done $=(0)$.

Levels of evaluation and selfevaluation-

Unsatisfactory $\geq 60 \%-75 \%$. -

Satisfactory $>75 \%-80 \%$.-

Excellent > $80 \%$

Tool II: Knowledge assessment of chest nursing care procedures steps, evaluation and self evaluation process (Appendix II). This tool developed by researcher to assess head nurses and nurses' knowledge of chest nursing care (six procedures) steps ,principles of performance evaluation and self evaluation , based on Lammogila (2013) (16), Walsh et al. (2007) ${ }^{(17)}$ and current relevant literatures. The tool include four parts as follows: Part one Identification data of both head nurses and nurses.

Part two Knowledge assessment of six chest nursing care standard procedures:- This part include questions on steps of standard nursing care of chest six procedures. The test include questions on Steps of chest care procedures standard performance consists of 7 questions (1-7). Indication of chest care procedures standard performance consists of 6 questions (8-13) Contraindication of chest care procedures standard performance consists of 7 questions (1420) Equipment required equipment of chest care procedures according standard performance consists of 3 questions (2123). Preparation of patient regarding chest care procedures performance standard consists of 9 questions ( 24-32).

Evaluate desirable and undesirable outcome 4 question (33-36) about assessment of desirable and undesirable outcome of care standard Infection control implementation of chest care 
procedures following infection control consists of 4 question (37-40) Part three Knowledge assessment for evaluation principles. This part include questions on evaluation process for its plan, needed information principles, methods and steps of implementation as follow: Plan consists of 5 questions (41-45) about evaluation plan process - Needed information 2 questions (46-47) about required information of evaluation process

- Principles 7 questions (48-54) about principles of evaluation process Methods and steps of implementation 7 questions ( 55-60) about methods and steps of evaluation process.

Part four Knowledge assessment on self-evaluation principles. This part include questions on self -evaluation process for its plan, needed information principles, methods and steps of implementation. As follow:

Plan consists of 5 questions (61-65) about self evaluation principles

- Needed information 2 questions (6667) about required information of selfevaluation principles

- Principles 9 questions ( 68-76) about principles of self-evaluation

-Methods and steps of implementation 6 questions ( 77-82) about methods and steps of implementation of selfevaluation to identify gap and treat

\section{Scoring system}

The correct answer take score (1) and incorrect answer take score (0).

\section{Level of knowledge will be:}

Good $=(>75 \%)$

Average $=(60-<75)$

Poor $=(>60 \%)$.

Method:1 -An official permission to conduct the study was obtained through litter from authorities at Faculty of nursing, Tanta University to director and nursing directors of hospitals under study.

Ethical Consideration Informed consent obtained from the head nurses and nurses after explaining the process of the study. Confidentiality of nursing staff relevant information is ascertained to them instead of nature of study and the right of withdrawal from the study is reserved. 2The tools (I,II) developed by researcher and tool (1) presented to seven experts in the administration and medical surgical area of specialty the experts were two lecturer and one assistant professors of nursing administration and one lecturer of and three assistant professors of medicalsurgical nursing at Faculty of Tanta and Moniefia universities. They were asked to evaluate tools relevance and appropriateness on 4 points rating scales 


\section{Tanta Scientific Nursing Journal}

as; $\quad$ not relevant $=1, \quad$ little relevant $=2$ ,relevant $=3$ and strongly relevant $=4$. Necessary modification were done included clarification, omission of certain question.

3- The opinions of the experts on tools (1) of the study were analyzed and determined the following; Tool 1 face validity ( $92 \%$ ) ,content validity index (CVI)100\% internal consistency reliability $(95 \%)$.

4- A pilot study was conducted on (10\%) of subjects to test the tools for clarity, and applicability then needed correction were done. The study was carried out two times on a sample of (2) head nurses and (8) staff nurses they randomly share from mentioned hospital and excluded from the sample.

The first time implemented after the development of the tools and second time implemented before starting the actual data collection to test the clarity of items and applicability and the relevance of the questions

The estimated time needed by head nurses and staff nurses to fill the scale was ( 20-30) minute for each sheet. 5 Reliability of tool (1) was tested use Croncbach's Alpha coefficient test. Its value was $(0.868)$.

Vol. 18 No. 1 May, 2020 


\section{Tanta Scientific Nursing Journal}

care procedures, evaluation and selfevaluation principles.

Learning strategies Appropriate learning strategies were used as demonstration, power point, group discussion ,lecture and hand out.

Implementation of the program. The study was carried on (20 head nurses ,80 nurses) The subject divided into six groups and the educational program include five sessions, every session (2hours) e.g (10 hours) for each group were conducted for nurses and head nurses at their work places of hospitals.

The subject informed about objective of the program and its sessions.

Evaluation the program -Tool (I) Pre, immediate and three month post implementation of educational program done on head nurses and nurses to assess actual performance of chest nursing care procedures regarding followed the standard steps. Tool (II) Pre, immediate and three month post implementation of educational chest nursing care program done to assess and evaluate changes of both head nurses and nurses level of knowledge about chest nursing care.

Statistical analysis of the data Data were fed to the computer and analyzed using IBM SPSS software package version 20.0. (Armonk, NY: IBM Corp)
Qualitative data were described using number and percent. Quantitative data were described using range (minimum and maximum), mean, standard deviation. Significance of the obtained results was judged at the 5\% level. The used tests ere 1 - Chi-square test For categorical variables, to compare between different groups .

\section{2 - Fisher's Exact or Monte Carlo} correction Correction for chi-square when more than $20 \%$ of the cells have expected count less than 5 .

3 - Friedman test For abnormally distributed quantitative variables, to compare between more than two periods or stages

Result: Table (1) Shows characteristics of subjects both head nurses and nurses including age, education level, years of experience, marital status, attendance previous training on evaluation, number and subject. The total subject $55 \%$ age ranged from 20- 30 years with mean $30.17 \pm 9.27$.Total subject $(72.0 \%)$ were married, 93\% not have training and $86.0 \%$ not have subject in evaluation except $35 \%$ of head nurses had subject in administration. Head nurses $55 \%$ their age ranged (41-50) with mean $40.90 \pm$ 4.27. All head nurses had B ScN and majority $(65 \%)$ have $10-<20$ years of 


\section{Tanta Scientific Nursing Journal}

experience. Majority (68.8\%) of nurses aged 20-30years with mean $27.49 \pm 8.18$ , 58.5\% had diploma degree with $<10$ years of experience .

Figure (1) Shows researcher evaluation of excellent level of nurses total performance of turning care procedures pre, immediately post and 3month post program. Preprogram none of nurses had excellent level of total performance. But changed to be majority immediate and three months post program showed excellent level of performance.

Table (2) shows researcher evaluation of nurse's excellent total performance of six chest care procedures pre, immediate, and 3 months post program. The table reveals statistically significant improvement of nurses excellent level of total performance of each chest care procedures $(\mathrm{p}<0.001 *)$. Equal percent $(1.3 \%)$ of nurses showed excellent level preprogram for performing turning, postural drainage and percussion procedures which improved to range $(96.3 \%-80 \%)$ post program and range $(98.8 \%-81.3 \%)$ 3month post program. While no one of nursing staff showed excellent level preprogram for vibration, coughing and suctioning procedures which changed post program to $65 \%, 78.8 \%$ and $100 \%$ and at 3 month post program to $65 \%, 83.3 \%$ and $100 \%$ respectively .

Table (3): Head nurses evaluation and nurses self -evaluation in level of performance of turning procedure items pre, post and 3 month post program. The table shows statistical significant improvement of head nurses evaluation and nurses self-evaluation of performance for all items of turning procedure at $(\mathbf{p}<\mathbf{0 . 0 0 1})$. Head nurses evaluation for preprogram showed that range (100\%-95\%)of nurses were at unsatisfactory level of performance for all items of turning procedure except $(60 \%)$ were at unsatisfactory level for item of preparing equipment. But post program nurses $95 \%$ were at excellent level for all items except for item of prepare patient by communication and teaching they were $85 \%$ and for item of following infection control they were $70 \%$.While at 3 month post program range (100-90\%)of nurses showed excellent performance for all items of procedure.

Nurses self- evaluation preprogram showed that range $(98.8-92.5 \%)$ were unsatisfactory perform all items of turning procedure except for item of implementation procedure by follow infection control they were $83.9 \%$.But 


\section{Tanta Scientific Nursing Journal}

post program range (91.3\%-85\%) were at excellent level except that at the items of following infection control they were $78.8 \%$.While post 3month program range (91.3-85\%) were at excellent level except for the item of following infection control they were $78.8 \%$.

Table (4): Head nurses evaluation and nurses self -evaluation of levels for performing postural drainage procedure items pre, post and 3 month post program. The table shows statistical significant improvement of head nurses evaluation and nurses self-evaluation of performing all items of postural drainage procedure at $(\mathrm{p}<0.001)$.

Pre -program head nurses evaluation for nurses performance of postural drainage procedures showed that nurses $100 \%$, $95 \%, 85 \%$ were at unsatisfactory level for assessment and contraindication and item of evaluate desirable and un desirable out come and prepare patient for teaching respectively .They $60 \%, 55 \%$ were at unsatisfactory level of performance items of preparation of equipment and implementation procedure following infection control respectively .But they $100-85 \%$ improved immediately post and $100-90 \%$ post 3 month were at excellent level for all item of the procedure.
Nurses self- evaluation preprogram showed that range $(98.8-86.3 \%)$ unsatisfactory perform all items of postural drainage procedure except $(67.5 \%)$ were for item preparation of equipment. But immediate post program range (92.5\%-81.3\%) were at excellent level except $(76.3 \%)$ they were at the items of implementation infection control .While 3 month post program range (100$85 \%$ ) were at excellent level except $(77.5 \%$ ) they were at the item of following infection control

Table (5) Head nurses evaluation and nurses self -evaluation of level of performing percussion procedure items pre, post and 3 month post program. The table shows statistical significant improvement of head nurses evaluation and nurses self-evaluation of performance for all items of percussion procedure at $(\mathrm{p}$ $<$ 0.001). Head nurses evaluation for preprogram showed that $(100 \%)$ of nurses were at unsatisfied level of performance for all items of percussion procedure except for implementing procedure following infection control only (65\%) were at unsatisfactory level.

But immediately post program 95\%-85\% of nurses were at excellent level for all items except for item of preparation patient by communication and teaching they were 


\section{Tanta Scientific Nursing Journal}

$75 \%$ and for item of prepare equipment they were $80 \%$.While 3 month post program equal percent (90\%)of nurses showed excellent performance for items of assessment indication and contraindication and items of evaluate desirable out- come. As well as items of prepare equipment and prepare patient by communication and teaching showed that equally percent $(80 \%)$ of nurses were at excellent level. Beside that all nurses showed excellent level in following infection control at implementing the procedure .

Nurses self- evaluation preprogram showed that $97.5,98.8 \%$ were at unsatisfactory level of performing at items of assessment indication and contraindication and evaluate desirable and undesirable out- come of percussion procedure .Equal percent $(77.5 \%)$ of nurses showed unsatisfactory level for performing item of implementation procedure by follow infection control and prepare equipment and $88.8 \%$ showed unsatisfactory at items of prepare patient equipment . But immediate post program nurses self -evaluation showed that 81.3 $\%$ of nurses showed excellent level of prepare patient communication and teaching. While range $(57.5 \%-66.3 \%)$ of nurses were at excellent level of rest items. Post 3month program nurses $83.8 \%, 70 \%$ perform implementation procedure following infection control and assessment indication ,contraindication of percussion, also range (67.5-57.5\%) perform items prepare patient by communication and teaching and evaluate desirable and undesirable out come at excellent level respectively .

Table (6): Head nurses evaluation and nurses self -evaluation of level of performing coughing exercise items of procedure pre, post and 3 month post program. The table shows statistical significant improvement of head nurses evaluation and nurses self-evaluation of performance for all items of coughing exercise procedure at $(\mathrm{p}<0.001)$. Head nurses evaluation for preprogram showed that all $(100 \%)$ of nurses were at unsatisfied level of performance for all items of coughing exercise procedure but changed post program nurses range (100\%-95\%) and at 3 month post program range (100-90\%) showed excellent performance for all items of coughing exercise procedure .

Nurses self- evaluation preprogram range (100\%-98.8\%) showed unsatisfactory level of performing all items of coughing exercise procedure except they were 


\section{Tanta Scientific Nursing Journal}

(21.3. \%) for item of preparation equipment . Post program range $(88.8 \%$ $75 \%$ ) were at excellent level for items of evaluate desirable and undesirable outcome, implementation procedure following infection control ,prepare equipment, prepare patient communication and teaching . Also nurses $68.8 \%$ of nurses showed excellent level at item of assessment indication and contraindication of coughing procedure at excellent level immediately post . 3month post program range (75 \%-82.5\%) were at excellent level for all items except they were $(91.3 \%)$ for item of evaluate desirable and undesirable out come .

Table (7): Head nurses evaluation and nurses self -evaluation of level for performing suctioning procedure items pre, post and 3 month post program. The table shows statistical significant improvement of head nurses evaluation and nurses self-evaluation of performance for all items of suctioning procedure at $(\mathrm{p}$ $<$ 0.001). Head nurses evaluation for preprogram showed that all $(100 \%)$ of nurses were at unsatisfied level of performance for all items of suctioning procedure except at item of prepare patient by communication and teaching they were (95\%) . But post program nurses range (100\%-90\%) and at 3 month post program range (100-90\%)of nurses showed excellent performance for all items of suctioning procedure

Nurses self- evaluation preprogram showed that nurses range (100\%-98.8\%) showed unsatisfactory perform once of all items of suctioning procedure changed post program to range ( $88.8-96.3 \%$ ) were at excellent level for all items except they were $(73.8 \%)$ for item of prepare patient by communication and teaching. While post 3month post program nurses range $(85 \%-97.5 \%)$ were at excellent level for performing all items of suctioning procedure .

Figure (2): represents Level of head nurse's and nurses overall knowledge about chest care procedures evaluation and selfevaluation pre, immediately post and 3 month post program. All head nurses preprogram showed poor level of knowledge changed post program to $45 \%$ and showed $90 \%$ of head nurses 3month post program poor level of knowledge .While $55 \%$ of head nurses showed at average level of total knowledge. All nurses preprogram showed poor level of knowledge changed post program to $60 \%$ showed average level of knowledge while $37.5 \%$ of nurses showed at good level of knowledge . 3month post program $45 \%$ of 


\section{Tanta Scientific Nursing Journal}

nurses poor level of knowledge . While $55 \%$ of nurses showed at average level of total knowledge respectively.

Table (8): represents Levels of head nurses total knowledge about chest care procedure, evaluation and self -evaluation principles pre, post and 3 month post program. The table shows statistical significance differences between head nurses knowledge of pre, post and 3 month for total knowledge for ,chest care evaluation and total knowledge at $(\mathrm{p}<0.001)$. Preprogram all head nurses showed poor level for all items of knowledge changed post program to $60 \%$, $70 \%$, showed good level knowledge about chest care procedures and evaluation principles respectively and all head nurses showed poor level knowledge about self evaluation principles pre and immediately post .Post 3 month program 85\%,95\% , $30 \%$ of head nurses showed poor level knowledge about chest care procedures , self-evaluation principles and evaluation principles respectively .

Table (9): represents Levels of nurses total knowledge about chest care procedure, evaluation and self -evaluation pre, post and 3 month post program. The table shows statistical significance differences between nurses knowledge of pre, post and 3 month for total knowledge for ,chest care evaluation and total knowledge at $\mathrm{p}<0.001$. Preprogram range (100\%-97.5\%) of nurses showed poor level for all items of knowledge changed post program to $73.8 \%, 71.3 \%$ and $21.3 \%$ showed good level post program .Post 3 month program $12.5 \%, 56.3 \%$ and $43.8 \%$ showed average level of knowledge respectively .

Table (10): Correlation between performing six chest nursing care procedures according to researcher's observation and nurses' self-evaluation of their performance pre, immediate post and 3 month post program . The table showed significant positive correlation at $(\mathrm{p}<0.001-\mathrm{p}<0.006)$ was detected in performing six chest nursing care procedures according to researcher's observation and nurses' self-evaluation. Table(11) Correlation between performing six chest nursing care procedures according to head nurse's observation and nurses' self-evaluation of their performance pre, immediate post \& 3 month post program. The table shows significant positive correlation at $\mathrm{P}<0.001$ in pre -program at turning procedure, postural drainage, percussion and suctioning, and at 3 month post program for percussion and suctioning procedures. 
Table (1) Characteristic of subjects both head nurses and nurses

\begin{tabular}{|c|c|c|c|c|c|c|}
\hline \multirow[t]{2}{*}{ Items } & \multicolumn{2}{|c|}{$\begin{array}{l}\text { Total head } \\
\text { nurses } \\
\text { \&nurses } \\
(\mathbf{n}=100) \\
\end{array}$} & \multicolumn{2}{|c|}{$\begin{array}{l}\text { Head nurses } \\
\quad(\mathbf{n}=\mathbf{2 0})\end{array}$} & \multicolumn{2}{|c|}{$\begin{array}{l}\text { Nurses } \\
(\mathbf{n}=\mathbf{8 0})\end{array}$} \\
\hline & No & $\%$ & No & $\%$ & No & $\%$ \\
\hline $\begin{array}{l}\text { Age (years) } \\
20-30 \\
31-40 \\
41-50 \\
\end{array}$ & $\begin{array}{l}55 \\
22 \\
23 \\
\end{array}$ & $\begin{array}{l}55.0 \\
22.0 \\
23.0\end{array}$ & $\begin{array}{c}0 \\
9 \\
11 \\
\end{array}$ & $\begin{array}{c}0.0 \\
45.0 \\
55.0\end{array}$ & $\begin{array}{l}55 \\
13 \\
12 \\
\end{array}$ & $\begin{array}{l}68.8 \\
16.3 \\
15.0\end{array}$ \\
\hline Mean \pm SD & \multicolumn{2}{|c|}{$30.17 \pm 9.27$} & \multicolumn{2}{|c|}{$40.90 \pm 4.27$} & \multicolumn{2}{|c|}{$27.49 \pm 8.18$} \\
\hline $\begin{array}{l}\text { Education level } \\
\text { Diploma } \\
\text { Technical institute } \\
\text { BScN } \\
\end{array}$ & $\begin{array}{l}47 \\
31 \\
22 \\
\end{array}$ & $\begin{array}{l}47.0 \\
31.0 \\
22.0\end{array}$ & $\begin{array}{c}0 \\
0 \\
20\end{array}$ & $\begin{array}{c}0.0 \\
0.0 \\
100.0\end{array}$ & $\begin{array}{c}47 \\
31 \\
2 \\
\end{array}$ & $\begin{array}{c}58.8 \\
38.8 \\
2.5 \\
\end{array}$ \\
\hline $\begin{array}{l}\text { Experience (years) in I.C.U } \\
<10 \\
10-<20 \\
20 \text { or more }\end{array}$ & $\begin{array}{l}60 \\
27 \\
13\end{array}$ & $\begin{array}{l}60.0 \\
27.0 \\
13.0\end{array}$ & $\begin{array}{c}0 \\
13 \\
7\end{array}$ & $\begin{array}{c}0.0 \\
65.0 \\
35.0\end{array}$ & $\begin{array}{c}60 \\
14 \\
6\end{array}$ & $\begin{array}{l}75.0 \\
17.5 \\
7.5\end{array}$ \\
\hline Mean \pm SD & \multicolumn{2}{|c|}{$8.31 \pm 8.04$} & \multicolumn{2}{|c|}{$18.45 \pm 3.99$} & \multicolumn{2}{|c|}{$5.78 \pm 6.68$} \\
\hline $\begin{array}{l}\text { Marital status } \\
\text { Married } \\
\text { Not married } \\
\text { Other } \\
\end{array}$ & $\begin{array}{c}72 \\
25 \\
3 \\
\end{array}$ & $\begin{array}{c}72.0 \\
25.0 \\
3.0 \\
\end{array}$ & $\begin{array}{c}18 \\
0 \\
2 \\
\end{array}$ & $\begin{array}{c}90.0 \\
0.0 \\
10.0 \\
\end{array}$ & $\begin{array}{c}54 \\
25 \\
1\end{array}$ & $\begin{array}{c}67.5 \\
31.3 \\
1.3 \\
\end{array}$ \\
\hline $\begin{array}{l}\text { Training on evaluation } \\
\text { No } \\
\text { Yes }\end{array}$ & $\begin{array}{c}93 \\
7\end{array}$ & $\begin{array}{c}93.0 \\
7.0\end{array}$ & $\begin{array}{c}13 \\
7 \\
\end{array}$ & $\begin{array}{l}65.0 \\
35.0\end{array}$ & $\begin{array}{c}80 \\
0\end{array}$ & $\begin{array}{c}100.0 \\
0.0\end{array}$ \\
\hline $\begin{array}{l}\text { Number of training } \\
\text { No } \\
\text { Once }\end{array}$ & $\begin{array}{c}93 \\
7 \\
\end{array}$ & $\begin{array}{c}93.0 \\
7.0\end{array}$ & $\begin{array}{c}13 \\
7 \\
\end{array}$ & $\begin{array}{l}65.0 \\
35.0 \\
\end{array}$ & $\begin{array}{c}80 \\
0\end{array}$ & $\begin{array}{c}100.0 \\
0.0\end{array}$ \\
\hline $\begin{array}{l}\text { Subjects in evaluation } \\
\text { No } \\
\text { Administration }\end{array}$ & $\begin{array}{l}86 \\
14\end{array}$ & $\begin{array}{l}86.0 \\
14.0\end{array}$ & $\begin{array}{c}13 \\
7\end{array}$ & $\begin{array}{l}65.0 \\
35.0\end{array}$ & $\begin{array}{c}73 \\
7\end{array}$ & $\begin{array}{c}91.3 \\
8.8\end{array}$ \\
\hline
\end{tabular}




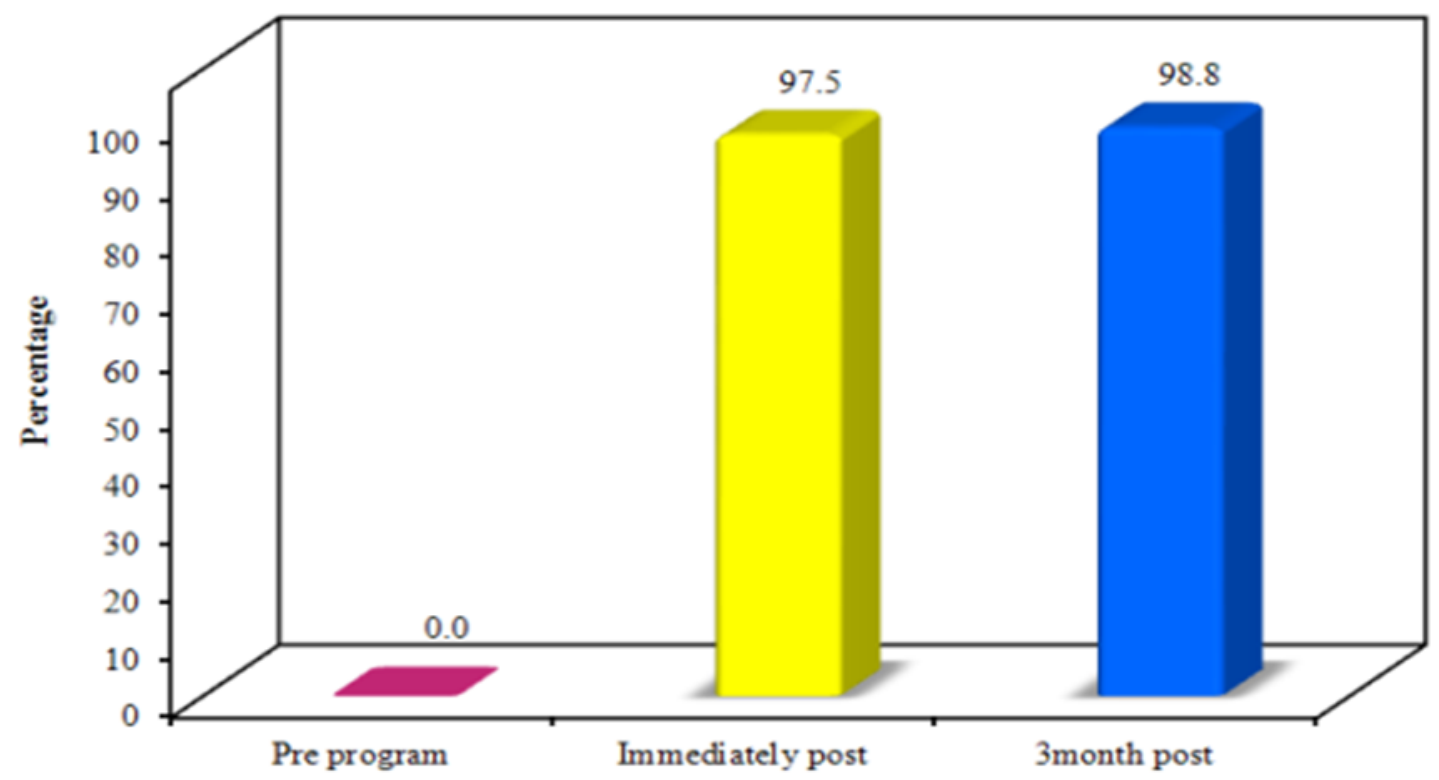

Figure (1): Researcher evaluation of excellent level of nurses' total performance of turning procedures pre, post 3 month post program $(n=80)$

\section{Researcher evaluation of nurse's performance of Six chest care procedures}

Table (2): Researcher evaluation of nurses excellent level of total performance of each chest care procedures $(n=80)$

\begin{tabular}{||l|c|c|c|c|c|c|c||}
\hline \multirow{2}{*}{ Chest care procedure } & \multicolumn{6}{|c|}{ Good level } & \multirow{2}{*}{} \\
\cline { 2 - 8 } & \multicolumn{2}{|c|}{$\begin{array}{c}\text { Pre } \\
\text { program }\end{array}$} & $\begin{array}{c}\text { Immediately } \\
\text { post }\end{array}$ & \multicolumn{2}{c|}{$\begin{array}{c}\text { 3month } \\
\text { post }\end{array}$} & \multirow{2}{*}{ p } \\
\cline { 2 - 8 } & No. & $\%$ & No. & $\%$ & No. & $\%$ & \\
\hline -Turning procedure & 1 & 1.3 & 77 & 96.3 & 79 & 98.8 & $<0.001^{*}$ \\
\hline $\begin{array}{l}\text {-Postural drainage } \\
\text { procedure }\end{array}$ & 1 & 1.3 & 66 & 82.5 & 71 & 88.8 & $<0.001^{*}$ \\
\hline -Percussion procedure & 1 & 1.3 & 64 & 80.0 & 65 & 81.3 & $<0.001^{*}$ \\
\hline -Vibration procedure & 0 & 0.0 & 52 & 65.0 & 52 & 65.0 & $<0.001^{*}$ \\
\hline $\begin{array}{l}\text {-Coughing exercise } \\
\text { procedure }\end{array}$ & 0 & 0.0 & 63 & 78.8 & 67 & 83.8 & $<0.001^{*}$ \\
\hline -Suctioning procedure & 0 & 0.0 & 80 & 100.0 & 80 & 100.0 & $<0.001^{*}$ \\
\hline
\end{tabular}




\section{Tanta Scientific Nursing Journal}

Table (3): Head nurses evaluation and nurses self -evaluation of performing turning procedure items pre, post and 3 month post program $(\mathrm{N}=20 \& 80)$

\begin{tabular}{|c|c|c|c|c|c|c|c|c|c|c|c|}
\hline \multirow{3}{*}{\multicolumn{2}{|c|}{ Turning procedure }} & \multicolumn{3}{|c|}{ pre program } & \multicolumn{3}{|c|}{ post program } & \multicolumn{3}{|c|}{3 month post program } & \multirow{3}{*}{ p1 } \\
\hline & & \multirow{2}{*}{\begin{tabular}{|c|} 
Excellent \\
$\begin{array}{c}\text { No. } \\
\%\end{array}$ \\
\end{tabular}} & \multirow{2}{*}{$\begin{array}{c}\text { Satisfactory } \\
\begin{array}{c}\text { No. } \\
\%\end{array} \\
\end{array}$} & \multirow{2}{*}{\begin{tabular}{|c|}
$\begin{array}{c}\text { Un } \\
\text { satisfactory }\end{array}$ \\
$\begin{array}{c}\text { No. } \\
\%\end{array}$ \\
\end{tabular}} & \multirow{2}{*}{\begin{tabular}{|c|} 
Excellent \\
$\begin{array}{c}\text { No. } \\
\%\end{array}$ \\
\end{tabular}} & \multirow{2}{*}{$\begin{array}{c}\text { Satisfactory } \\
\begin{array}{c}\text { No. } \\
\%\end{array}\end{array}$} & \multirow{2}{*}{$\begin{array}{c}\begin{array}{c}\text { Un } \\
\text { satisfactory }\end{array} \\
\begin{array}{c}\text { No. } \\
\%\end{array} \\
\end{array}$} & \multirow{2}{*}{$\begin{array}{c}\text { Excellent } \\
\begin{array}{c}\text { No. } \\
\%\end{array} \\
\end{array}$} & \multirow{2}{*}{\begin{tabular}{c|} 
Satisfactor \\
$\underset{\%}{N o .}$ \\
$\%$
\end{tabular}} & \multirow{2}{*}{$\begin{array}{c}\begin{array}{c}\text { Un } \\
\text { satisfactory }\end{array} \\
\begin{array}{c}\text { No. } \\
\%\end{array} \\
\end{array}$} & \\
\hline & & & & & & & & & & & \\
\hline \multirow{2}{*}{$\begin{array}{c}\text { Assessment } \\
\text { indication } \\
\text { turning }\end{array}$} & $\begin{array}{c}\text { Head nurse } \\
\text { evaluation }\end{array}$ & $\begin{array}{c}0 \\
0.0\end{array}$ & $\begin{array}{c}0 \\
0.0\end{array}$ & $\begin{array}{c}20 \\
100.0 \\
\end{array}$ & $\begin{array}{c}19 \\
95.0 \\
\end{array}$ & $\begin{array}{c}1 \\
5.0 \\
\end{array}$ & $\begin{array}{c}0 \\
0.0 \\
\end{array}$ & $\begin{array}{c}20 \\
100.0 \\
\end{array}$ & $\begin{array}{c}0 \\
0.0 \\
\end{array}$ & $\begin{array}{c}0 \\
0.0 \\
\end{array}$ & $<0.001^{*}$ \\
\hline & $\begin{array}{c}\text { Nurses self - } \\
\text { evaluation }\end{array}$ & $\begin{array}{c}1 \\
1.3 \\
\end{array}$ & $\begin{array}{c}0 \\
0.0 \\
\end{array}$ & $\begin{array}{c}79 \\
98.8 \\
\end{array}$ & $\begin{array}{c}68 \\
85.0 \\
\end{array}$ & $\begin{array}{c}10 \\
12.5 \\
\end{array}$ & $\begin{array}{c}2 \\
2.5\end{array}$ & $\begin{array}{c}68 \\
85.0 \\
\end{array}$ & $\begin{array}{c}10 \\
12.5 \\
\end{array}$ & $\begin{array}{c}2 \\
2.5 \\
\end{array}$ & $<0.001^{*}$ \\
\hline \multicolumn{2}{|c|}{$\chi^{2}(\mathbf{p})$} & \multicolumn{3}{|c|}{$0.253\left({ }^{\mathrm{FE}} \mathrm{p}=1.000\right)$} & \multicolumn{3}{|c|}{$0.922\left({ }^{\mathrm{MC}} \mathrm{p}=0.651\right)$} & \multicolumn{3}{|c|}{$2.839\left({ }^{\mathrm{MC}} \mathrm{p}=0.229\right)$} & \\
\hline \multirow{2}{*}{$\begin{array}{c}\text { Preparation } \\
\text { equipment }\end{array}$} & \begin{tabular}{|c} 
Head nurse \\
evaluation
\end{tabular} & $\begin{array}{c}8 \\
40.0 \\
\end{array}$ & $\begin{array}{c}0 \\
0.0\end{array}$ & $\begin{array}{c}12 \\
60.0 \\
\end{array}$ & $\begin{array}{c}19 \\
95.0\end{array}$ & $\begin{array}{c}0 \\
0.0\end{array}$ & $\begin{array}{c}1 \\
5.0\end{array}$ & $\begin{array}{c}19 \\
95.0\end{array}$ & $\begin{array}{c}0 \\
0.0\end{array}$ & $\begin{array}{c}1 \\
5.0\end{array}$ & $<0.001^{*}$ \\
\hline & $\begin{array}{c}\text { Nurses self - } \\
\text { evaluation }\end{array}$ & $\begin{array}{c}6 \\
7.5\end{array}$ & $\begin{array}{c}0 \\
0.0\end{array}$ & $\begin{array}{c}74 \\
92.5 \\
\end{array}$ & $\begin{array}{c}70 \\
87.5\end{array}$ & $\begin{array}{c}0 \\
0.0\end{array}$ & $\begin{array}{c}10 \\
12.5\end{array}$ & $\begin{array}{c}73 \\
91.3 \\
\end{array}$ & $\begin{array}{c}0 \\
0.0\end{array}$ & $\begin{array}{c}7 \\
8.8 \\
\end{array}$ & $<0.001^{*}$ \\
\hline \multicolumn{2}{|c|}{$\chi 2($ FEp) } & \multicolumn{3}{|c|}{$14.037^{*}\left(0.001^{*}\right)$} & \multicolumn{3}{|c|}{$0.919(0.456)$} & \multicolumn{3}{|c|}{$0.306(1.000)$} & \\
\hline \multirow{2}{*}{$\begin{array}{c}\text { Prepare } \\
\text { patient } \\
\text { communicatio }\end{array}$} & $\begin{array}{c}\text { Head nurse } \\
\text { evaluation }\end{array}$ & $\begin{array}{c}0 \\
0.0\end{array}$ & $\begin{array}{c}1 \\
5.0\end{array}$ & $\begin{array}{c}19 \\
95.0\end{array}$ & $\begin{array}{c}17 \\
85.0\end{array}$ & $\begin{array}{c}3 \\
15.0\end{array}$ & $\begin{array}{c}0 \\
0.0\end{array}$ & $\begin{array}{c}19 \\
95.0\end{array}$ & $\begin{array}{c}1 \\
5.0\end{array}$ & $\begin{array}{c}0 \\
0.0\end{array}$ & $<0.001^{*}$ \\
\hline & \begin{tabular}{|c} 
Nurses self - \\
evaluation
\end{tabular} & $\begin{array}{c}2 \\
2.5 \\
\end{array}$ & $\begin{array}{c}4 \\
5.0 \\
\end{array}$ & $\begin{array}{c}74 \\
92.5 \\
\end{array}$ & $\begin{array}{c}68 \\
85.0 \\
\end{array}$ & $\begin{array}{c}11 \\
13.8 \\
\end{array}$ & $\begin{array}{c}1 \\
1.3 \\
\end{array}$ & $\begin{array}{c}72 \\
90.0 \\
\end{array}$ & $\begin{array}{c}8 \\
10.0 \\
\end{array}$ & $\begin{array}{c}0 \\
0.0\end{array}$ & $<0.001^{*}$ \\
\hline \multicolumn{2}{|c|}{$\chi^{2}(\mathbf{p})$} & \multicolumn{3}{|c|}{$0.392\left({ }^{\mathrm{MC}} \mathrm{p}=1.000\right)$} & \multicolumn{3}{|c|}{$0.570\left({ }^{\mathrm{MC}} \mathrm{p}=1.000\right)$} & \multicolumn{3}{|c|}{$0.488\left({ }^{\mathrm{FE}} \mathrm{p}=0.683\right)$} & \\
\hline \multirow{2}{*}{$\begin{array}{c}\text { Implementati } \\
\text { following } \\
\text { infection } \\
\text { control }\end{array}$} & $\begin{array}{c}\text { Head nurse } \\
\text { evaluation }\end{array}$ & $\begin{array}{c}0 \\
0.0\end{array}$ & $\begin{array}{c}0 \\
0.0\end{array}$ & $\begin{array}{c}20 \\
100.0\end{array}$ & $\begin{array}{c}14 \\
70.0\end{array}$ & $\begin{array}{c}5 \\
25.0\end{array}$ & $\begin{array}{c}1 \\
5.0\end{array}$ & $\begin{array}{c}18 \\
90.0\end{array}$ & $\begin{array}{c}2 \\
10.0\end{array}$ & $\begin{array}{c}0 \\
0.0\end{array}$ & $<0.001^{*}$ \\
\hline & $\begin{array}{c}\begin{array}{c}\text { Nurses self - } \\
\text { evaluation }\end{array} \\
\end{array}$ & $\begin{array}{c}6 \\
7.5\end{array}$ & $\begin{array}{c}7 \\
8.8\end{array}$ & $\begin{array}{c}67 \\
83.8\end{array}$ & $\begin{array}{c}63 \\
78.8 \\
\end{array}$ & $\begin{array}{c}14 \\
17.5 \\
\end{array}$ & $\begin{array}{c}3 \\
3.8 \\
\end{array}$ & $\begin{array}{c}56 \\
70.0 \\
\end{array}$ & $\begin{array}{c}19 \\
23.8 \\
\end{array}$ & $\begin{array}{c}5 \\
6.3 \\
\end{array}$ & $<0.001^{*}$ \\
\hline \multicolumn{2}{|c|}{$\chi^{2}\left({ }^{\mathrm{MC}} \mathrm{p}\right)$} & \multicolumn{3}{|c|}{$2.624(0.215)$} & \multicolumn{3}{|c|}{$1.158(0.529)$} & \multicolumn{3}{|c|}{$2.754(0.243)$} & \\
\hline \begin{tabular}{|l|} 
Evaluate \\
desirable
\end{tabular} & $\begin{array}{c}\text { Head nurse } \\
\text { evaluation }\end{array}$ & \begin{tabular}{|c|}
0 \\
0.0 \\
\end{tabular} & $\begin{array}{l}0 \\
0.0\end{array}$ & $\begin{array}{c}20 \\
100.0\end{array}$ & \begin{tabular}{|c|}
19 \\
95.0
\end{tabular} & $\begin{array}{c}1 \\
5.0\end{array}$ & $\begin{array}{c}0 \\
0.0\end{array}$ & $\begin{array}{c}19 \\
95.0\end{array}$ & $\begin{array}{c}1 \\
5.0\end{array}$ & \begin{tabular}{|c|}
0 \\
0.0
\end{tabular} & $<0.001^{*}$ \\
\hline $\begin{array}{c}\text { \&undesirable } \\
\text { outcome }\end{array}$ & $\begin{array}{c}\text { Nurses self - } \\
\text { evaluation }\end{array}$ & \begin{tabular}{|c|}
0 \\
0.0 \\
\end{tabular} & $\begin{array}{l}1 \\
1.3 \\
\end{array}$ & $\begin{array}{c}79 \\
98.8 \\
\end{array}$ & \begin{tabular}{|c|}
73 \\
91.3 \\
\end{tabular} & $\begin{array}{c}6 \\
7.5 \\
\end{array}$ & $\begin{array}{c}1 \\
1.3 \\
\end{array}$ & $\begin{array}{c}73 \\
91.3 \\
\end{array}$ & $\begin{array}{c}6 \\
7.5 \\
\end{array}$ & $\begin{array}{c}1 \\
1.3 \\
\end{array}$ & $<0.001^{*}$ \\
\hline & & & $3\left({ }^{\mathrm{FE}} \mathrm{p}=\right.$ & $.000)$ & & $3\left({ }^{\mathrm{MC}}{ }_{\mathrm{p}}\right.$ & 00) & 0.543 & $\left({ }^{\mathrm{MC}} \mathrm{p}=1\right.$. & $.000)$ & \\
\hline
\end{tabular}

$\chi^{2}$ : Chi square test MC: Monte Carlo FE: Fisher Exact p: $p$ value for comparison between head nurses evaluation and nurses in different period $p_{1}: p$ value for Friedman test comparing between the studied periods *: Statistically significant at $p \leq 0.05$ 


\section{Tanta Scientific Nursing Journal}

Table (4): Head nurses evaluation and nurses self -evaluation of level for performing of postural drainage procedures items pre, post and 3 month post program $(\mathrm{N}=20 \& 80)$

\begin{tabular}{|c|c|c|c|c|c|c|c|c|c|c|c|}
\hline \multirow{3}{*}{\multicolumn{2}{|c|}{$\begin{array}{c}\text { Postural drainage } \\
\text { procedure }\end{array}$}} & \multicolumn{3}{|c|}{ pre program } & \multicolumn{3}{|c|}{ post program } & \multicolumn{3}{|c|}{3 month post program } & \multirow{3}{*}{$\mathrm{p}_{1}$} \\
\hline & & \multirow{2}{*}{\begin{tabular}{|c|} 
Excellent \\
No. \\
$\%$
\end{tabular}} & \multirow{2}{*}{\begin{tabular}{|c|} 
Satisfactory \\
No. \\
$\%$
\end{tabular}} & \multirow{2}{*}{\begin{tabular}{|c|}
$\begin{array}{c}\text { Un } \\
\text { satisfactory }\end{array}$ \\
$\begin{array}{c}\text { No. } \\
\%\end{array}$ \\
\end{tabular}} & \multirow{2}{*}{\begin{tabular}{|c|} 
Excellent \\
No. \\
$\%$
\end{tabular}} & \multirow{2}{*}{\begin{tabular}{|c|} 
Satisfactory \\
No. \\
$\%$
\end{tabular}} & \multirow{2}{*}{\begin{tabular}{|c|}
$\begin{array}{c}\text { Un } \\
\text { satisfactory }\end{array}$ \\
$\begin{array}{c}\text { No. } \\
\%\end{array}$ \\
\end{tabular}} & \multirow{2}{*}{$\begin{array}{c}\text { Excellent } \\
\begin{array}{c}\text { No. } \\
\%\end{array}\end{array}$} & \multirow{2}{*}{\begin{tabular}{|c|} 
Satisfactory \\
$\begin{array}{c}\text { No. } \\
\%\end{array}$ \\
\end{tabular}} & \multirow{2}{*}{\begin{tabular}{|c|}
$\begin{array}{c}\text { Un } \\
\text { Satisfacto }\end{array}$ \\
No. \\
$\%$ \\
\end{tabular}} & \\
\hline & & & & & & & & & & & \\
\hline \multirow{2}{*}{$\begin{array}{l}\text { Assessment } \\
\text { indication } \\
\text {,contraindicat }\end{array}$} & $\begin{array}{c}\text { Head nurse } \\
\text { evaluation }\end{array}$ & $\begin{array}{c}0 \\
0.0\end{array}$ & $\begin{array}{c}0 \\
0.0\end{array}$ & $\begin{array}{c}20 \\
100.0\end{array}$ & $\begin{array}{c}19 \\
95.0\end{array}$ & $\begin{array}{c}1 \\
5.0\end{array}$ & $\begin{array}{c}0 \\
0.0\end{array}$ & $\begin{array}{c}20 \\
100.0\end{array}$ & $\begin{array}{c}0 \\
0.0\end{array}$ & $\begin{array}{c}0 \\
0.0\end{array}$ & $\underset{*}{<0.001}$ \\
\hline & $\begin{array}{c}\text { Nurses self - } \\
\text { evaluation }\end{array}$ & $\begin{array}{c}1 \\
1.3 \\
\end{array}$ & $\begin{array}{c}0 \\
0.0 \\
\end{array}$ & $\begin{array}{c}79 \\
98.8 \\
\end{array}$ & $\begin{array}{c}65 \\
81.3 \\
\end{array}$ & $\begin{array}{c}10 \\
12.5 \\
\end{array}$ & $\begin{array}{c}5 \\
6.3 \\
\end{array}$ & $\begin{array}{c}69 \\
86.3 \\
\end{array}$ & $\begin{array}{c}6 \\
7.5 \\
\end{array}$ & $\begin{array}{c}5 \\
6.3 \\
\end{array}$ & $<0.001$ \\
\hline \multicolumn{2}{|l|}{$\chi^{2}(\mathbf{p})$} & \multicolumn{3}{|c|}{$0.253\left({ }^{\mathrm{FE}} \mathrm{p}=1.000\right)$} & \multicolumn{3}{|c|}{$1.539\left({ }^{\mathrm{MC}} \mathrm{p}=0.497\right)$} & \multicolumn{3}{|c|}{$1.913\left({ }^{\mathrm{MC}} \mathrm{p}=0.323\right)$} & \\
\hline \multirow{2}{*}{ Preparatioequipment } & $\begin{array}{c}\text { Head nurse } \\
\text { evaluation }\end{array}$ & $\begin{array}{c}8 \\
40.0 \\
\end{array}$ & $\begin{array}{c}0 \\
0.0\end{array}$ & $\begin{array}{c}12 \\
60.0\end{array}$ & $\begin{array}{c}17 \\
85.0\end{array}$ & $\begin{array}{c}0 \\
0.0\end{array}$ & $\begin{array}{c}3 \\
15.0 \\
\end{array}$ & $\begin{array}{c}19 \\
95.0\end{array}$ & $\begin{array}{c}0 \\
0.0\end{array}$ & $\begin{array}{c}1 \\
5.0\end{array}$ & $<\underset{*}{<001}$ \\
\hline & $\begin{array}{c}\text { Nurses self - } \\
\text { evaluation }\end{array}$ & $\begin{array}{c}26 \\
32.5 \\
\end{array}$ & $\begin{array}{c}0 \\
0.0\end{array}$ & $\begin{array}{c}54 \\
67.5 \\
\end{array}$ & $\begin{array}{c}73 \\
91.3 \\
\end{array}$ & $\begin{array}{c}0 \\
0.0\end{array}$ & $\begin{array}{c}7 \\
8.8 \\
\end{array}$ & $\begin{array}{c}73 \\
91.3 \\
\end{array}$ & $\begin{array}{c}0 \\
0.0\end{array}$ & $\begin{array}{c}7 \\
8.8 \\
\end{array}$ & $<0.001$ \\
\hline \multicolumn{2}{|l|}{$\chi^{2}(\mathbf{p})$} & \multicolumn{3}{|c|}{$0.401(0.527)$} & \multicolumn{3}{|c|}{$0.694\left({ }^{\mathrm{FE}} \mathrm{p}=0.414\right)$} & \multicolumn{3}{|c|}{$0.306\left({ }^{\mathrm{FE}} \mathrm{p}=1.000\right)$} & \\
\hline \multirow{2}{*}{$\begin{array}{l}\text { Prepare } \\
\text { patient } \\
\text { teaching }\end{array}$} & $\begin{array}{c}\text { Head nurse } \\
\text { evaluation }\end{array}$ & $\begin{array}{c}3 \\
15.0 \\
\end{array}$ & $\begin{array}{c}0 \\
0.0\end{array}$ & $\begin{array}{c}17 \\
85.0\end{array}$ & $\begin{array}{c}19 \\
95.0\end{array}$ & $\begin{array}{c}0 \\
0.0\end{array}$ & $\begin{array}{c}1 \\
5.0\end{array}$ & $\begin{array}{c}20 \\
100.0\end{array}$ & $\begin{array}{c}0 \\
0.0\end{array}$ & $\begin{array}{c}0 \\
0.0\end{array}$ & $<0.001$ \\
\hline & $\begin{array}{c}\text { Nurses self } \\
- \\
\text { evaluation }\end{array}$ & $\begin{array}{c}11 \\
13.8\end{array}$ & $\begin{array}{c}0 \\
0.0\end{array}$ & $\begin{array}{c}69 \\
86.3\end{array}$ & $\begin{array}{c}74 \\
92.5\end{array}$ & $\begin{array}{c}0 \\
0.0\end{array}$ & $\begin{array}{c}6 \\
7.5\end{array}$ & $\begin{array}{c}80 \\
100.0\end{array}$ & $\begin{array}{c}0 \\
0.0\end{array}$ & $\begin{array}{c}0 \\
0.0\end{array}$ & $<0.001$ \\
\hline \multicolumn{2}{|l|}{$\chi^{2}(\mathbf{p})$} & \multicolumn{3}{|c|}{$0.021\left({ }^{F E} p=1.000\right)$} & \multicolumn{3}{|c|}{$0.154\left({ }^{F E} p=1.000\right)$} & \multicolumn{3}{|c|}{ - } & \\
\hline \multirow{2}{*}{$\begin{array}{l}\text { Implement } \\
\text { procedure } \\
\text { following } \\
\text { infection } \\
\text { control } \\
\end{array}$} & $\begin{array}{c}\text { Head nurse } \\
\text { evaluation }\end{array}$ & $\begin{array}{c}0 \\
0.0\end{array}$ & $\begin{array}{c}9 \\
45.0\end{array}$ & $\begin{array}{c}11 \\
55.0\end{array}$ & $\begin{array}{c}20 \\
100.0\end{array}$ & $\begin{array}{c}0 \\
0.0\end{array}$ & $\begin{array}{c}0 \\
0.0\end{array}$ & $\begin{array}{c}19 \\
95.0\end{array}$ & $\begin{array}{c}1 \\
5.0\end{array}$ & $\begin{array}{c}0 \\
0.0\end{array}$ & $<\underset{*}{<001}$ \\
\hline & \begin{tabular}{|c|}
$\begin{array}{c}\text { Nurses self - } \\
\text { evaluation }\end{array}$ \\
\end{tabular} & $\begin{array}{c}0 \\
0.0 \\
\end{array}$ & $\begin{array}{c}1 \\
1.3 \\
\end{array}$ & $\begin{array}{c}79 \\
98.8 \\
\end{array}$ & $\begin{array}{c}61 \\
76.3 \\
\end{array}$ & $\begin{array}{c}14 \\
17.5 \\
\end{array}$ & $\begin{array}{c}5 \\
6.3 \\
\end{array}$ & $\begin{array}{c}62 \\
77.5 \\
\end{array}$ & $\begin{array}{c}17 \\
21.3 \\
\end{array}$ & $\begin{array}{c}1 \\
1.3 \\
\end{array}$ & $<0.001$ \\
\hline \multicolumn{2}{|l|}{$\chi^{2}(\mathbf{p})$} & \multicolumn{3}{|c|}{ 34.028 $\left.^{*(\mathrm{FE}} \mathbf{p}<0.001^{*}\right)$} & \multicolumn{3}{|c|}{$5.427^{*}\left({ }^{\mathrm{MC}} \mathrm{p}=0.045^{*}\right)$} & \multicolumn{3}{|c|}{$3.267\left({ }^{\mathrm{MC}} \mathrm{p}=0.233\right)$} & \\
\hline & $\begin{array}{c}\text { Head nurse } \\
\text { evaluation }\end{array}$ & $\begin{array}{c}0 \\
0.0\end{array}$ & $\begin{array}{c}1 \\
5.0\end{array}$ & $\begin{array}{c}19 \\
95.0\end{array}$ & $\begin{array}{c}18 \\
90.0\end{array}$ & $\begin{array}{c}2 \\
10.0\end{array}$ & $\begin{array}{c}0 \\
0.0\end{array}$ & $\begin{array}{c}18 \\
90.0\end{array}$ & $\begin{array}{c}2 \\
10.0\end{array}$ & $\begin{array}{c}0 \\
0.0\end{array}$ & $<\underset{*}{<001}$ \\
\hline $\begin{array}{l}\text { undesirable } \\
\text { outcome }\end{array}$ & \begin{tabular}{|c|}
$\begin{array}{c}\text { Nurses self - } \\
\text { evaluation }\end{array}$ \\
\end{tabular} & $\begin{array}{c}3 \\
3.8 \\
\end{array}$ & $\begin{array}{c}6 \\
7.5 \\
\end{array}$ & $\begin{array}{c}71 \\
88.8 \\
\end{array}$ & $\begin{array}{c}65 \\
81.3 \\
\end{array}$ & $\begin{array}{c}10 \\
12.5 \\
\end{array}$ & $\begin{array}{c}5 \\
6.3 \\
\end{array}$ & $\begin{array}{c}68 \\
85.0 \\
\end{array}$ & $\begin{array}{c}6 \\
7.5 \\
\end{array}$ & $\begin{array}{c}6 \\
7.5 \\
\end{array}$ & $<0.001$ \\
\hline$\chi^{2}$ & & & $98\left({ }^{\mathrm{MC}} \mathrm{p}\right.$ & $000)$ & 0.85 & $\left({ }^{\mathrm{MC}} \mathrm{p}=\right.$ & & 1.3 & ${ }^{\mathrm{MC}} \mathrm{p}_{\mathrm{p}}$ & 60) & \\
\hline
\end{tabular}

$\chi^{2}$ : Chi square test MC: Monte Carlo FE: Fisher Exact p: $p$ value for comparison between head nurses evaluation and nurses in different period $p_{1}$ : $p$ value for Friedman test comparing between the studied periods *: Statistically significant at $\mathbf{p} \leq \mathbf{0 . 0 5}$ 


\section{Tanta Scientific Nursing Journal}

Table (5): Head nurses evaluation and nurses self -evaluation for performing percussion procedure items pre, post and 3 month post program $(\mathrm{N}=20 \& 80)$

\begin{tabular}{|c|c|c|c|c|c|c|c|c|c|c|c|}
\hline \multirow{2}{*}{\multicolumn{2}{|c|}{ Percussion procedure }} & \multicolumn{3}{|c|}{ pre program } & \multicolumn{3}{|c|}{ post program } & \multicolumn{3}{|c|}{3 month post program } & \multirow{3}{*}{$\mathbf{p}_{1}$} \\
\hline & & \multirow{2}{*}{\begin{tabular}{c|} 
Excellent \\
$\begin{array}{c}\text { No. } \\
\%\end{array}$ \\
\end{tabular}} & \multirow{2}{*}{\begin{tabular}{|c|} 
Satisfactory \\
$\begin{array}{c}\text { No. } \\
\%\end{array}$
\end{tabular}} & \multirow{2}{*}{$\begin{array}{c}\begin{array}{c}\text { Un } \\
\text { satisfactory }\end{array} \\
\begin{array}{c}\text { No. } \\
\%\end{array}\end{array}$} & \multirow{2}{*}{\begin{tabular}{|c|} 
Excellent \\
$\begin{array}{c}\text { No. } \\
\%\end{array}$
\end{tabular}} & \multirow{2}{*}{\begin{tabular}{c|} 
Satisfactory \\
No. \\
$\%$
\end{tabular}} & \multirow{2}{*}{\begin{tabular}{|c|}
$\begin{array}{c}\text { Un } \\
\text { satisfactory }\end{array}$ \\
$\begin{array}{c}\text { No. } \\
\%\end{array}$ \\
\end{tabular}} & \multirow{2}{*}{$\begin{array}{c}\text { Excellent } \\
\begin{array}{c}\text { No. } \\
\%\end{array}\end{array}$} & \multirow{2}{*}{$\begin{array}{c}\text { Satisfactory } \\
\begin{array}{c}\text { No. } \\
\%\end{array}\end{array}$} & \multirow{2}{*}{\begin{tabular}{|c|}
$\begin{array}{c}\text { Un } \\
\text { satisfactory }\end{array}$ \\
$\begin{array}{c}\text { No. } \\
\%\end{array}$ \\
\end{tabular}} & \\
\hline & & & & & & & & & & & \\
\hline $\begin{array}{l}\text { Assessment } \\
\text { indication }\end{array}$ & \begin{tabular}{|l|}
$\begin{array}{l}\text { Head nurse } \\
\text { evaluation }\end{array}$ \\
\end{tabular} & $\begin{array}{c}0 \\
0.0 \\
\end{array}$ & $\begin{array}{c}0 \\
0.0\end{array}$ & $\begin{array}{c}20 \\
100.0 \\
\end{array}$ & $\begin{array}{c}17 \\
85.0 \\
\end{array}$ & $\begin{array}{c}2 \\
10.0 \\
\end{array}$ & $\begin{array}{c}1 \\
5.0 \\
\end{array}$ & $\begin{array}{c}18 \\
90.0 \\
\end{array}$ & $\begin{array}{c}2 \\
10.0 \\
\end{array}$ & $\begin{array}{c}0 \\
0.0 \\
\end{array}$ & $<0.001^{*}$ \\
\hline $\begin{array}{l}\text {,contraindication } \\
\text { percussion }\end{array}$ & $\begin{array}{l}\text { Nurses self } \\
\text {-evaluation }\end{array}$ & $\begin{array}{c}0 \\
0.0\end{array}$ & $\begin{array}{c}2 \\
2.5\end{array}$ & $\begin{array}{c}78 \\
97.5\end{array}$ & $\begin{array}{c}52 \\
65.0\end{array}$ & $\begin{array}{c}14 \\
17.5\end{array}$ & $\begin{array}{c}14 \\
17.5\end{array}$ & $\begin{array}{c}56 \\
70.0\end{array}$ & $\begin{array}{c}15 \\
18.8\end{array}$ & $\begin{array}{c}9 \\
11.3\end{array}$ & $<0.001^{*}$ \\
\hline \multicolumn{2}{|l|}{$\chi^{2}(\mathrm{p})$} & \multicolumn{3}{|c|}{$0.510\left({ }^{F E} p=1.000\right)$} & \multicolumn{3}{|c|}{$2.701\left({ }^{\mathrm{MC}} \mathrm{p}=0.254\right)$} & \multicolumn{3}{|c|}{$3.247\left({ }^{\mathrm{MC}} \mathrm{p}=0.192\right)$} & \\
\hline \multirow{2}{*}{$\begin{array}{l}\text { Preparation } \\
\text { equipment }\end{array}$} & \begin{tabular}{|c|} 
Head nurse \\
evaluation
\end{tabular} & $\begin{array}{c}0 \\
0.0\end{array}$ & $\begin{array}{c}0 \\
0.0\end{array}$ & $\begin{array}{c}20 \\
100.0\end{array}$ & $\begin{array}{c}16 \\
80.0\end{array}$ & $\begin{array}{c}1 \\
5.0\end{array}$ & $\begin{array}{c}3 \\
15.0\end{array}$ & $\begin{array}{c}16 \\
80.0\end{array}$ & $\begin{array}{c}1 \\
5.0\end{array}$ & $\begin{array}{c}3 \\
15.0\end{array}$ & $<0.001^{*}$ \\
\hline & \begin{tabular}{|l|} 
Nurses self \\
-evaluation
\end{tabular} & $\begin{array}{c}6 \\
7.5 \\
\end{array}$ & $\begin{array}{c}12 \\
15.0 \\
\end{array}$ & $\begin{array}{c}62 \\
77.5 \\
\end{array}$ & $\begin{array}{c}53 \\
66.3 \\
\end{array}$ & $\begin{array}{c}15 \\
18.8 \\
\end{array}$ & $\begin{array}{c}12 \\
15.0 \\
\end{array}$ & $\begin{array}{c}53 \\
66.3 \\
\end{array}$ & $\begin{array}{c}15 \\
18.8 \\
\end{array}$ & $\begin{array}{c}12 \\
15.0 \\
\end{array}$ & $<0.001^{*}$ \\
\hline \multicolumn{2}{|l|}{$\chi^{2}(\mathrm{MCp})$} & \multicolumn{3}{|c|}{$4.827(0.070)$} & \multicolumn{3}{|c|}{$2.180(0.348)$} & \multicolumn{3}{|c|}{$2.180(0.348)$} & \\
\hline \multirow{2}{*}{$\begin{array}{l}\text { Prepare patient } \\
\text { communication } \\
\& \text { teaching }\end{array}$} & $\begin{array}{c}\text { Head nurse } \\
\text { evaluation }\end{array}$ & $\begin{array}{c}0 \\
0.0\end{array}$ & $\begin{array}{c}0 \\
0.0\end{array}$ & $\begin{array}{c}20 \\
100.0\end{array}$ & $\begin{array}{c}15 \\
75.0\end{array}$ & $\begin{array}{c}5 \\
25.0\end{array}$ & $\begin{array}{c}0 \\
0.0\end{array}$ & $\begin{array}{c}16 \\
80.0\end{array}$ & $\begin{array}{c}4 \\
20.0\end{array}$ & $\begin{array}{c}0 \\
0.0\end{array}$ & $<0.001^{*}$ \\
\hline & \begin{tabular}{|l|} 
Nurses self \\
-evaluation
\end{tabular} & $\begin{array}{c}1 \\
1.3 \\
\end{array}$ & $\begin{array}{c}8 \\
10.0 \\
\end{array}$ & $\begin{array}{c}71 \\
88.8 \\
\end{array}$ & $\begin{array}{c}51 \\
63.8 \\
\end{array}$ & $\begin{array}{c}24 \\
30.0 \\
\end{array}$ & $\begin{array}{c}5 \\
6.3 \\
\end{array}$ & $\begin{array}{c}54 \\
67.5 \\
\end{array}$ & $\begin{array}{c}23 \\
28.8 \\
\end{array}$ & $\begin{array}{c}3 \\
3.8 \\
\end{array}$ & $<0.001^{*}$ \\
\hline \multicolumn{2}{|l|}{$\chi^{2}\left({ }^{\mathrm{MC}} \mathrm{p}\right)$} & \multicolumn{3}{|c|}{$2.213(0.479)$} & \multicolumn{3}{|c|}{$1.073(0.624)$} & \multicolumn{3}{|c|}{$0.973(0.695)$} & \\
\hline \multirow{2}{*}{$\begin{array}{l}\text { Implementation } \\
\text { following } \\
\text { infection control }\end{array}$} & $\begin{array}{c}\text { Head nurse } \\
\text { evaluation }\end{array}$ & $\begin{array}{c}0 \\
0.0 \\
\end{array}$ & $\begin{array}{c}7 \\
35.0\end{array}$ & $\begin{array}{c}13 \\
65.0\end{array}$ & $\begin{array}{c}19 \\
95.0\end{array}$ & $\begin{array}{c}1 \\
5.0\end{array}$ & $\begin{array}{c}0 \\
0.0\end{array}$ & $\begin{array}{c}20 \\
100.0\end{array}$ & $\begin{array}{c}0 \\
0.0\end{array}$ & $\begin{array}{c}0 \\
0.0\end{array}$ & $<0.001^{*}$ \\
\hline & \begin{tabular}{|l|} 
Nurses self \\
-evaluation
\end{tabular} & $\begin{array}{c}0 \\
0.0 \\
\end{array}$ & $\begin{array}{c}18 \\
22.5 \\
\end{array}$ & $\begin{array}{c}62 \\
77.5 \\
\end{array}$ & $\begin{array}{c}65 \\
81.3 \\
\end{array}$ & $\begin{array}{c}15 \\
18.8 \\
\end{array}$ & $\begin{array}{c}0 \\
0.0 \\
\end{array}$ & $\begin{array}{c}67 \\
83.8 \\
\end{array}$ & $\begin{array}{c}12 \\
15.0 \\
\end{array}$ & $\begin{array}{c}1 \\
1.3 \\
\end{array}$ & $<0.001^{*}$ \\
\hline \multicolumn{2}{|l|}{$\chi^{2}(\mathrm{p})$} & \multicolumn{3}{|c|}{$1.333(0.248)$} & \multicolumn{3}{|c|}{$2.251\left({ }^{\mathrm{FE}} \mathrm{p}=0.183\right)$} & \multicolumn{3}{|c|}{$3.876\left({ }^{\mathrm{MC}}=0.187\right)$} & \\
\hline \multirow{2}{*}{$\begin{array}{l}\text { Evaluate } \\
\text { desirable } \\
\text { undesirable } \\
\text { outcome }\end{array}$} & \begin{tabular}{|c|} 
Head nurse \\
evaluation
\end{tabular} & $\begin{array}{c}0 \\
0.0\end{array}$ & $\begin{array}{c}0 \\
0.0\end{array}$ & $\begin{array}{c}20 \\
100.0\end{array}$ & $\begin{array}{c}18 \\
90.0\end{array}$ & $\begin{array}{c}2 \\
10.0\end{array}$ & $\begin{array}{c}0 \\
0.0\end{array}$ & $\begin{array}{c}18 \\
90.0\end{array}$ & $\begin{array}{c}2 \\
10.0\end{array}$ & $\begin{array}{c}0 \\
0.0\end{array}$ & $<0.001^{*}$ \\
\hline & \begin{tabular}{|l|} 
Nurses self \\
-evaluation
\end{tabular} & $\begin{array}{c}0 \\
0.0\end{array}$ & $\begin{array}{c}1 \\
1.3\end{array}$ & $\begin{array}{c}79 \\
98.8\end{array}$ & $\begin{array}{c}46 \\
57.5\end{array}$ & $\begin{array}{c}30 \\
37.5 \\
\end{array}$ & $\begin{array}{c}4 \\
5.0\end{array}$ & $\begin{array}{c}46 \\
57.5\end{array}$ & $\begin{array}{c}30 \\
37.5 \\
\end{array}$ & $\begin{array}{c}4 \\
5.0\end{array}$ & $<0.001^{*}$ \\
\hline$\chi^{2}(\mathbf{p})$ & & & $53\left({ }^{\mathrm{FE}} \mathrm{p}=1\right.$ & 00) & 6.99 & $1^{*}\left({ }^{M C} p=0\right.$ & $\left.025^{*}\right)$ & 6.99 & ${ }^{*}\left({ }^{\mathrm{MC}} \mathrm{p}=\mathbf{0}\right.$. & $\left.025^{*}\right)$ & \\
\hline
\end{tabular}

Table (6): Head nurses evaluation and nurses self -evaluation of level for performing coughing exercise items pre, post and 3 month post program $(\mathrm{N}=20 \& 80)$ 


\section{Tanta Scientific Nursing Journal}

\begin{tabular}{|c|c|c|c|c|c|c|c|c|c|c|c|}
\hline \multirow{2}{*}{\multicolumn{2}{|c|}{ Coughing exercise }} & \multicolumn{3}{|c|}{ pre program } & \multicolumn{3}{|c|}{ post program } & \multicolumn{3}{|c|}{3 month post program } & \multirow{3}{*}{$\mathbf{p}_{1}$} \\
\hline & & \multirow{2}{*}{\begin{tabular}{|c|} 
Excellent \\
$\begin{array}{c}\text { No. } \\
\%\end{array}$ \\
\end{tabular}} & \multirow{2}{*}{$\begin{array}{c}\text { Satisfactory } \\
\begin{array}{c}\text { No. } \\
\%\end{array}\end{array}$} & \multirow{2}{*}{\begin{tabular}{|c|}
$\begin{array}{c}\text { Un } \\
\text { satisfactory }\end{array}$ \\
$\begin{array}{c}\text { No. } \\
\text { \% }\end{array}$
\end{tabular}} & \multirow{2}{*}{\begin{tabular}{|c|} 
Excellent \\
$\begin{array}{c}\text { No. } \\
\%\end{array}$ \\
\end{tabular}} & \multirow{2}{*}{$\begin{array}{c}\text { Satisfactory } \\
\begin{array}{c}\text { No. } \\
\%\end{array}\end{array}$} & \multirow{2}{*}{\begin{tabular}{|c|}
$\begin{array}{c}\text { Un } \\
\text { satisfactory }\end{array}$ \\
$\begin{array}{c}\text { No. } \\
\text { \% }\end{array}$
\end{tabular}} & \multirow{2}{*}{\begin{tabular}{|c|} 
Excellent \\
$\begin{array}{c}\text { No. } \\
\%\end{array}$
\end{tabular}} & \multirow{2}{*}{\begin{tabular}{|c|} 
Satisfactory \\
$\begin{array}{c}\text { No. } \\
\%\end{array}$ \\
\end{tabular}} & \multirow{2}{*}{\begin{tabular}{|c|}
$\begin{array}{c}\text { Un } \\
\text { satisfactory }\end{array}$ \\
$\begin{array}{c}\text { No. } \\
\%\end{array}$ \\
\end{tabular}} & \\
\hline & & & & & & & & & & & \\
\hline \multirow{2}{*}{\begin{tabular}{|l} 
Assessment \\
indication \\
,contraindica \\
coughing
\end{tabular}} & $\begin{array}{l}\begin{array}{l}\text { Head nurse } \\
\text { evaluation }\end{array} \\
\end{array}$ & $\begin{array}{c}0 \\
0.0\end{array}$ & $\begin{array}{c}0 \\
0.0\end{array}$ & $\begin{array}{c}20 \\
100.0\end{array}$ & $\begin{array}{c}20 \\
100.0\end{array}$ & $\begin{array}{c}0 \\
0.0\end{array}$ & $\begin{array}{c}0 \\
0.0\end{array}$ & $\begin{array}{c}20 \\
100.0\end{array}$ & $\begin{array}{c}0 \\
0.0\end{array}$ & $\begin{array}{c}0 \\
0.0\end{array}$ & $<\underset{*}{<0.001}$ \\
\hline & $\begin{array}{c}\text { Nurses self - } \\
\text { evaluation }\end{array}$ & $\begin{array}{c}0 \\
0.0\end{array}$ & $\begin{array}{c}0 \\
0.0\end{array}$ & $\begin{array}{c}80 \\
100.0\end{array}$ & $\begin{array}{c}55 \\
68.8 \\
\end{array}$ & $\begin{array}{c}6 \\
7.5\end{array}$ & $\begin{array}{c}19 \\
23.8\end{array}$ & $\begin{array}{c}60 \\
75.0\end{array}$ & $\begin{array}{c}7 \\
8.8\end{array}$ & $\begin{array}{c}13 \\
16.3\end{array}$ & $<0.001$ \\
\hline \multicolumn{2}{|c|}{$\chi^{2}(\mathrm{p})$} & \multicolumn{3}{|c|}{-} & \multicolumn{3}{|c|}{$8.547^{*}\left({ }^{\mathrm{MC}} \mathrm{p}=0.009^{*}\right)$} & \multicolumn{3}{|c|}{$5.744^{*}\left({ }^{\mathrm{MC}} \mathrm{p}=0.044^{*}\right)$} & \\
\hline \multirow{2}{*}{$\begin{array}{l}\text { Preparation } \\
\text { equipment }\end{array}$} & \begin{tabular}{|c} 
Head nurse \\
evaluation
\end{tabular} & \begin{tabular}{|c|}
20 \\
100.0
\end{tabular} & $\begin{array}{c}0 \\
0.0\end{array}$ & $\begin{array}{c}0 \\
0.0\end{array}$ & $\begin{array}{c}20 \\
100.0\end{array}$ & $\begin{array}{c}0 \\
0.0\end{array}$ & $\begin{array}{c}0 \\
0.0\end{array}$ & $\begin{array}{c}20 \\
100.0\end{array}$ & $\begin{array}{c}0 \\
0.0\end{array}$ & $\begin{array}{c}0 \\
0.0\end{array}$ & - \\
\hline & \begin{tabular}{|c|}
$\begin{array}{c}\text { Nurses self - } \\
\text { evaluation }\end{array}$ \\
\end{tabular} & $\begin{array}{c}63 \\
78.8 \\
\end{array}$ & $\begin{array}{c}0 \\
0.0 \\
\end{array}$ & $\begin{array}{c}17 \\
21.3 \\
\end{array}$ & $\begin{array}{c}63 \\
78.8 \\
\end{array}$ & $\begin{array}{c}0 \\
0.0 \\
0.0\end{array}$ & $\begin{array}{c}17 \\
21.3 \\
\end{array}$ & $\begin{array}{c}63 \\
78.8 \\
\end{array}$ & $\begin{array}{c}0 \\
0.0\end{array}$ & $\begin{array}{c}17 \\
21.3 \\
\end{array}$ & - \\
\hline \multicolumn{2}{|c|}{$\chi^{2}\left({ }^{\mathrm{FE}} \mathrm{p}\right)$} & \multicolumn{3}{|c|}{$5.120^{*}\left(0.020^{*}\right)$} & \multicolumn{3}{|c|}{$5.120^{*}\left(0.020^{*}\right)$} & \multicolumn{3}{|c|}{$5.120^{*}\left(0.020^{*}\right)$} & \\
\hline \multirow{2}{*}{$\begin{array}{l}\text { Prepare patient } \\
\text { communicatite } \\
\text { aching }\end{array}$} & \begin{tabular}{|l|} 
Head nurse \\
evaluation
\end{tabular} & $\begin{array}{c}0 \\
0.0\end{array}$ & $\begin{array}{c}0 \\
0.0\end{array}$ & $\begin{array}{c}20 \\
100.0\end{array}$ & $\begin{array}{l}18 \\
90.0\end{array}$ & $\begin{array}{c}2 \\
10.0\end{array}$ & $\begin{array}{c}0 \\
0.0\end{array}$ & $\begin{array}{l}18 \\
90.0\end{array}$ & $\begin{array}{c}2 \\
10.0\end{array}$ & $\begin{array}{c}0 \\
0.0\end{array}$ & $<0.001$ \\
\hline & \begin{tabular}{|c|}
$\begin{array}{c}\text { Nurses self } \\
\text { evaluation }\end{array}$ \\
\end{tabular} & $\begin{array}{c}1 \\
1.3\end{array}$ & $\begin{array}{c}0 \\
0.0\end{array}$ & $\begin{array}{c}79 \\
98.8\end{array}$ & $\begin{array}{c}60 \\
75.0 \\
\end{array}$ & $\begin{array}{c}18 \\
22.5\end{array}$ & $\begin{array}{c}2 \\
2.5 \\
\end{array}$ & $\begin{array}{c}60 \\
75.0 \\
\end{array}$ & $\begin{array}{c}18 \\
22.5 \\
\end{array}$ & $\begin{array}{c}2 \\
2.5\end{array}$ & $<\underset{*}{<001}$ \\
\hline \multicolumn{2}{|c|}{$\chi^{2}(p)$} & \multicolumn{3}{|c|}{$0.253\left({ }^{\mathrm{FE}} \mathrm{p}=1.000\right)$} & \multicolumn{3}{|c|}{$1.720\left({ }^{\mathrm{MC}} \mathrm{p}=0.497\right)$} & \multicolumn{3}{|c|}{$1.720\left({ }^{\mathrm{MC}} \mathrm{p}=0.497\right)$} & \\
\hline \multirow{2}{*}{\begin{tabular}{|l|} 
Implementatpr \\
ocedure \\
following \\
infection \\
control \\
\end{tabular}} & $\begin{array}{c}\text { Head nurse } \\
\text { evaluation }\end{array}$ & $\begin{array}{c}0 \\
0.0\end{array}$ & $\begin{array}{c}0 \\
0.0\end{array}$ & $\begin{array}{c}20 \\
100.0 \\
\end{array}$ & $\begin{array}{c}19 \\
95.0\end{array}$ & $\begin{array}{c}1 \\
5.0\end{array}$ & $\begin{array}{c}0 \\
0.0\end{array}$ & $\begin{array}{c}20 \\
100.0\end{array}$ & $\begin{array}{c}0 \\
0.0\end{array}$ & $\begin{array}{c}0 \\
0.0\end{array}$ & $<0.001$ \\
\hline & \begin{tabular}{|c|}
$\begin{array}{c}\text { Nurses self - } \\
\text { evaluation }\end{array}$ \\
\end{tabular} & $\begin{array}{c}0 \\
0.0\end{array}$ & $\begin{array}{c}1 \\
1.3 \\
\end{array}$ & $\begin{array}{c}79 \\
98.8\end{array}$ & $\begin{array}{c}66 \\
82.5 \\
\end{array}$ & $\begin{array}{c}9 \\
11.3 \\
\end{array}$ & $\begin{array}{c}5 \\
6.3 \\
\end{array}$ & $\begin{array}{c}66 \\
82.5 \\
\end{array}$ & $\begin{array}{c}9 \\
11.3 \\
\end{array}$ & $\begin{array}{c}5 \\
6.3 \\
\end{array}$ & $<\underset{*}{<0.001}$ \\
\hline \multicolumn{2}{|c|}{$\chi^{2}(\mathrm{p})$} & \multicolumn{3}{|c|}{$0.253\left({ }^{\mathrm{FE}} \mathrm{p}=1.000\right)$} & \multicolumn{3}{|c|}{$1.307\left({ }^{\mathrm{MC}} \mathrm{p}=0.562\right)$} & \multicolumn{3}{|c|}{$3.089\left({ }^{M C} p=0.183\right)$} & \\
\hline $\begin{array}{l}\text { Evaluate } \\
\text { desirable }\end{array}$ & \begin{tabular}{|c|}
$\begin{array}{c}\text { Head nurse } \\
\text { evaluation }\end{array}$ \\
\end{tabular} & $\begin{array}{c}0 \\
0.0\end{array}$ & $\begin{array}{c}0 \\
0.0\end{array}$ & $\begin{array}{c}20 \\
100.0 \\
\end{array}$ & $\begin{array}{c}19 \\
95.0\end{array}$ & $\begin{array}{c}1 \\
5.0 \\
\end{array}$ & $\begin{array}{c}0 \\
0.0\end{array}$ & $\begin{array}{c}20 \\
100.0 \\
\end{array}$ & $\begin{array}{c}0 \\
0.0 \\
\end{array}$ & $\begin{array}{c}0 \\
0.0 \\
\end{array}$ & $<0.001$ \\
\hline $\begin{array}{l}\text { undesirable } \\
\text { outcome }\end{array}$ & \begin{tabular}{|c|}
$\begin{array}{c}\text { Nurses self - } \\
\text { evaluation }\end{array}$ \\
\end{tabular} & $\begin{array}{c}0 \\
0.0\end{array}$ & $\begin{array}{c}0 \\
0.0\end{array}$ & $\begin{array}{c}80 \\
100.0\end{array}$ & $\begin{array}{c}71 \\
88.8\end{array}$ & $\begin{array}{c}6 \\
7.5\end{array}$ & $\begin{array}{c}3 \\
3.8\end{array}$ & $\begin{array}{c}73 \\
91.3\end{array}$ & $\begin{array}{c}7 \\
8.8\end{array}$ & $\begin{array}{c}0 \\
0.0\end{array}$ & $<0.001$ \\
\hline$\chi^{2}$ & & & - & & 0.3 & $8\left({ }^{\mathrm{MC}} \mathrm{p}=1\right.$ & $.000)$ & 1.8 & $82\left({ }^{\mathrm{FE}} \mathrm{p}=0\right.$ & 339) & \\
\hline
\end{tabular}

Table (7 ): Head nurses evaluation and nurses self -evaluation of level for performing suctioning procedure items pre, post \& 3 month post program(N=100\& 80$)$ 


\section{Tanta Scientific Nursing Journal}

\begin{tabular}{|c|c|c|c|c|c|c|c|c|c|c|c|}
\hline \multirow{3}{*}{\multicolumn{2}{|c|}{ Suctioning procedure }} & \multicolumn{3}{|c|}{ pre program } & \multicolumn{3}{|c|}{ post program } & \multicolumn{3}{|c|}{3 month post program } & \multirow{3}{*}{$\mathbf{p}_{1}$} \\
\hline & & \multirow{2}{*}{\begin{tabular}{|c|} 
Excellent \\
$\begin{array}{c}\text { No. } \\
\%\end{array}$ \\
\end{tabular}} & \multirow{2}{*}{\begin{tabular}{|c|}
$\begin{array}{c}\text { Satisfact } \\
\text { ory }\end{array}$ \\
$\begin{array}{c}\text { No. } \\
\%\end{array}$ \\
\end{tabular}} & \multirow{2}{*}{\begin{tabular}{|c|}
$\begin{array}{c}\text { Un } \\
\text { satisfact } \\
\text { ory }\end{array}$ \\
$\begin{array}{c}\text { No. } \\
\%\end{array}$ \\
\end{tabular}} & \multirow{2}{*}{\begin{tabular}{|c|}
$\begin{array}{c}\text { Excellen } \\
\mathbf{t}\end{array}$ \\
$\begin{array}{c}\text { No. } \\
\%\end{array}$ \\
\end{tabular}} & \multirow{2}{*}{\begin{tabular}{|c|}
$\begin{array}{c}\text { Satisfact } \\
\text { ory }\end{array}$ \\
$\begin{array}{c}\text { No. } \\
\%\end{array}$ \\
\end{tabular}} & \multirow{2}{*}{\begin{tabular}{|c|}
$\begin{array}{c}\text { Un } \\
\text { satisfactor } \\
\text { y }\end{array}$ \\
$\begin{array}{c}\text { No. } \\
\%\end{array}$ \\
\end{tabular}} & \multirow{2}{*}{\begin{tabular}{|c|}
$\begin{array}{c}\text { Excellen } \\
\mathbf{t}\end{array}$ \\
$\begin{array}{c}\text { No. } \\
\%\end{array}$ \\
\end{tabular}} & \multirow{2}{*}{$\begin{array}{c}\text { Satisfactor } \\
\mathbf{y} \\
\begin{array}{c}\text { No. } \\
\%\end{array} \\
\end{array}$} & \multirow{2}{*}{\begin{tabular}{|c|c}
$\begin{array}{c}\text { Un } \\
\text { satisfactor } \\
\mathbf{y}\end{array}$ \\
$\begin{array}{c}\text { No. } \\
\%\end{array}$ \\
\end{tabular}} & \\
\hline & & & & & & & & & & & \\
\hline Assessment & $\begin{array}{c}\text { Head nurse } \\
\text { evaluation }\end{array}$ & $\begin{array}{c}0 \\
0.0\end{array}$ & $\begin{array}{c}0 \\
0.0\end{array}$ & $\begin{array}{c}20 \\
100.0\end{array}$ & $\begin{array}{c}20 \\
100.0\end{array}$ & $\begin{array}{c}0 \\
0.0\end{array}$ & $\begin{array}{c}0 \\
0.0\end{array}$ & $\begin{array}{c}20 \\
100.0\end{array}$ & $\begin{array}{c}0 \\
0.0\end{array}$ & $\begin{array}{c}0 \\
0.0\end{array}$ & $<0.001^{*}$ \\
\hline $\begin{array}{l}\text {,contraindication } \\
\text { of suction }\end{array}$ & $\begin{array}{c}\text { Nurses self - } \\
\text { evaluation }\end{array}$ & $\begin{array}{c}0 \\
0.0\end{array}$ & $\begin{array}{c}0 \\
0.0\end{array}$ & $\begin{array}{c}80 \\
100.0\end{array}$ & $\begin{array}{c}76 \\
95.0\end{array}$ & $\begin{array}{c}3 \\
3.8\end{array}$ & $\begin{array}{c}1 \\
1.3\end{array}$ & $\begin{array}{c}76 \\
95.0\end{array}$ & $\begin{array}{c}3 \\
3.8\end{array}$ & $\begin{array}{c}1 \\
1.3\end{array}$ & $<0.001^{*}$ \\
\hline \multicolumn{2}{|l|}{$\chi^{2}(\mathbf{p})$} & \multicolumn{3}{|c|}{-} & \multicolumn{3}{|c|}{$0.748\left({ }^{\mathrm{MC}} \mathrm{p}=1.000\right)$} & \multicolumn{3}{|c|}{$0.748\left({ }^{\mathrm{MC}} \mathrm{p}=1.000\right)$} & \\
\hline \multirow{2}{*}{$\begin{array}{l}\text { Preparation } \\
\text { equipment }\end{array}$} & \begin{tabular}{|c|} 
Head nurse \\
evaluation
\end{tabular} & $\begin{array}{c}0 \\
0.0\end{array}$ & $\begin{array}{c}0 \\
0.0\end{array}$ & $\begin{array}{c}20 \\
100.0\end{array}$ & $\begin{array}{c}20 \\
100.0\end{array}$ & $\begin{array}{c}0 \\
0.0\end{array}$ & $\begin{array}{c}0 \\
0.0\end{array}$ & $\begin{array}{c}20 \\
100.0\end{array}$ & $\begin{array}{c}0 \\
0.0\end{array}$ & $\begin{array}{c}0 \\
0.0\end{array}$ & $<0.001^{*}$ \\
\hline & \begin{tabular}{|c|} 
Nurses self - \\
evaluation
\end{tabular} & $\begin{array}{c}0 \\
0.0 \\
\end{array}$ & $\begin{array}{c}1 \\
1.3 \\
\end{array}$ & $\begin{array}{c}79 \\
98.8 \\
\end{array}$ & $\begin{array}{c}71 \\
88.8 \\
\end{array}$ & $\begin{array}{c}9 \\
11.3 \\
\end{array}$ & $\begin{array}{c}0 \\
0.0 \\
\end{array}$ & $\begin{array}{l}76 \\
95.0 \\
\end{array}$ & $\begin{array}{c}4 \\
5.0 \\
\end{array}$ & $\begin{array}{c}0 \\
0.0 \\
\end{array}$ & $<0.001^{*}$ \\
\hline \multicolumn{2}{|l|}{$\chi^{2}\left({ }^{\mathrm{FE}} \mathrm{p}\right)$} & \multicolumn{3}{|c|}{$0.253(1.000)$} & \multicolumn{3}{|c|}{$2.473(0.198)$} & \multicolumn{3}{|c|}{$1.042(0.581)$} & \\
\hline \multirow{2}{*}{$\begin{array}{l}\text { Prepare patient } \\
\text { by } \\
\text { communication \& } \\
\text { teaching }\end{array}$} & $\begin{array}{c}\text { Head nurse } \\
\text { evaluation }\end{array}$ & $\begin{array}{c}0 \\
0.0\end{array}$ & $\begin{array}{c}1 \\
5.0\end{array}$ & $\begin{array}{c}19 \\
95.0\end{array}$ & $\begin{array}{c}18 \\
90.0\end{array}$ & $\begin{array}{c}2 \\
10.0\end{array}$ & $\begin{array}{c}0 \\
0.0\end{array}$ & $\begin{array}{c}18 \\
90.0\end{array}$ & $\begin{array}{c}1 \\
5.0\end{array}$ & $\begin{array}{c}1 \\
5.0\end{array}$ & $<0.001^{*}$ \\
\hline & $\begin{array}{c}\text { Nurses self } \\
\text {-evaluation }\end{array}$ & $\begin{array}{c}0 \\
0.0\end{array}$ & $\begin{array}{c}1 \\
1.3 \\
\end{array}$ & $\begin{array}{c}79 \\
98.8 \\
\end{array}$ & $\begin{array}{c}59 \\
73.8 \\
\end{array}$ & $\begin{array}{c}19 \\
23.8 \\
\end{array}$ & $\begin{array}{c}2 \\
2.5\end{array}$ & $\begin{array}{c}68 \\
85.0\end{array}$ & $\begin{array}{c}10 \\
12.5 \\
\end{array}$ & $\begin{array}{c}2 \\
2.5\end{array}$ & $<0.001^{*}$ \\
\hline \multicolumn{2}{|l|}{$\chi^{2}(\mathbf{p})$} & \multicolumn{3}{|c|}{$1.148\left({ }^{\mathrm{FE}} \mathrm{p}=0.362\right)$} & \multicolumn{3}{|c|}{$2.009\left({ }^{\mathrm{MC}} \mathrm{p}=0.354\right)$} & \multicolumn{3}{|c|}{$1.374\left({ }^{\mathrm{MC}} \mathrm{p}=0.478\right)$} & \\
\hline \multirow{2}{*}{$\begin{array}{l}\text { Implementation } \\
\text { procedure } \\
\text { following } \\
\text { infection control }\end{array}$} & $\begin{array}{c}\text { Head nurse } \\
\text { evaluation }\end{array}$ & $\begin{array}{c}0 \\
0.0\end{array}$ & $\begin{array}{c}0 \\
0.0\end{array}$ & $\begin{array}{c}20 \\
100.0\end{array}$ & $\begin{array}{c}20 \\
100.0\end{array}$ & $\begin{array}{c}0 \\
0.0\end{array}$ & $\begin{array}{c}0 \\
0.0\end{array}$ & $\begin{array}{c}20 \\
100.0\end{array}$ & $\begin{array}{c}0 \\
0.0\end{array}$ & $\begin{array}{c}0 \\
0.0\end{array}$ & $<0.001^{*}$ \\
\hline & \begin{tabular}{|c|}
$\begin{array}{c}\text { Nurses self }- \\
\text { evaluation }\end{array}$ \\
\end{tabular} & $\begin{array}{c}0 \\
0.0 \\
\end{array}$ & $\begin{array}{c}0 \\
0.0 \\
\end{array}$ & $\begin{array}{c}80 \\
100.0 \\
\end{array}$ & $\begin{array}{c}77 \\
96.3 \\
\end{array}$ & $\begin{array}{c}3 \\
3.8 \\
\end{array}$ & $\begin{array}{c}0 \\
0.0\end{array}$ & $\begin{array}{c}78 \\
97.5 \\
\end{array}$ & $\begin{array}{c}2 \\
2.5 \\
\end{array}$ & $\begin{array}{c}0 \\
0.0\end{array}$ & $<0.001^{*}$ \\
\hline \multicolumn{2}{|l|}{$\chi^{2}(\mathbf{p})$} & \multicolumn{3}{|c|}{-} & \multicolumn{3}{|c|}{$0.773\left({ }^{\mathrm{FE}} \mathrm{p}=1.000\right)$} & \multicolumn{3}{|c|}{$0.510\left({ }^{\mathrm{FE}} \mathrm{p}=1.000\right)$} & \\
\hline \multirow{2}{*}{$\begin{array}{l}\text { Evaluate } \\
\text { desirable } \\
\text { undesirable } \\
\text { outcome }\end{array}$} & $\begin{array}{c}\text { Head nurse } \\
\text { evaluation }\end{array}$ & $\begin{array}{c}0 \\
0.0\end{array}$ & $\begin{array}{c}0 \\
0.0\end{array}$ & $\begin{array}{c}20 \\
100.0\end{array}$ & $\begin{array}{c}20 \\
100.0\end{array}$ & $\begin{array}{c}0 \\
0.0\end{array}$ & $\begin{array}{c}0 \\
0.0\end{array}$ & $\begin{array}{c}20 \\
100.0\end{array}$ & $\begin{array}{c}0 \\
0.0\end{array}$ & $\begin{array}{c}0 \\
0.0\end{array}$ & $<0.001^{*}$ \\
\hline & $\begin{array}{l}\text { Nurses self - } \\
\text { evaluatio }\end{array}$ & $\begin{array}{c}0 \\
0.0\end{array}$ & $\begin{array}{c}0 \\
0.0\end{array}$ & $\begin{array}{c}80 \\
100.0\end{array}$ & $\begin{array}{c}72 \\
90.0\end{array}$ & $\begin{array}{c}7 \\
8.8\end{array}$ & $\begin{array}{c}1 \\
1.3\end{array}$ & $\begin{array}{c}75 \\
93.8\end{array}$ & $\begin{array}{c}5 \\
6.3\end{array}$ & $\begin{array}{c}0 \\
0.0\end{array}$ & $<0.001^{*}$ \\
\hline$\chi^{2}(\mathbf{p})$ & & & - & & 1.8 & $46\left({ }^{\mathrm{MC}} \mathrm{p}=0\right.$ & $.471)$ & & $316\left({ }^{\mathrm{FE}} \mathrm{p}=0\right.$ & $580)$ & $\chi^{2}(\mathbf{p})$ \\
\hline
\end{tabular}




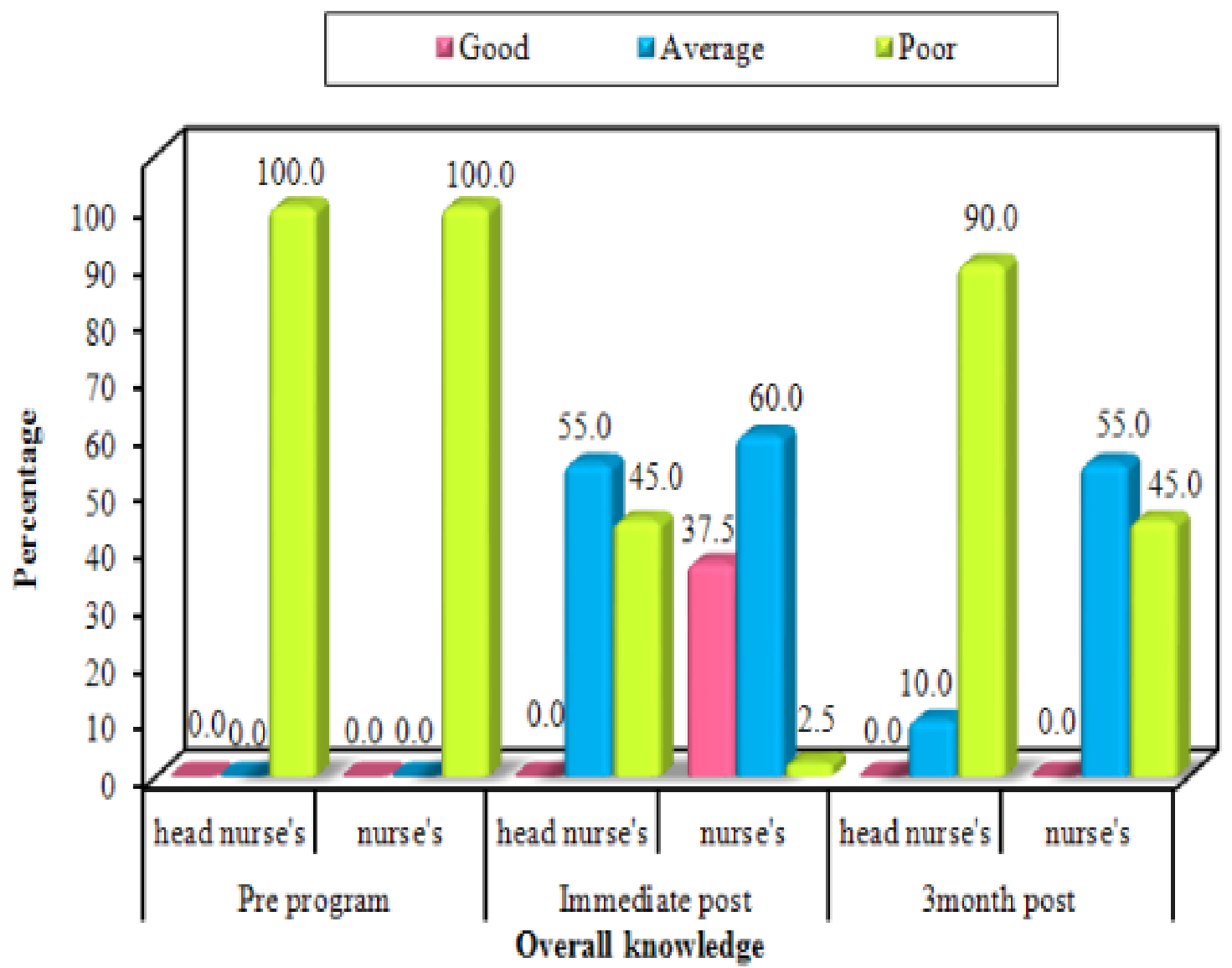

Figure (2) Level of head nurses and nurses overall knowledge about chest care procedures evaluation and self- evaluation pre, post and three month post program

Table (8): Levels of head nurse's total knowledge about chest care procedure, evaluation and self evaluation principles items $(\mathrm{N}=20)$

\begin{tabular}{|c|c|c|c|c|c|c|c|c|c|c|}
\hline \multirow{3}{*}{ Knowledge items } & \multicolumn{3}{|c|}{ Pre program } & \multicolumn{3}{|c|}{$\begin{array}{c}\text { Immediate post } \\
\text { program }\end{array}$} & \multicolumn{3}{|c|}{ Post program } & \multirow{3}{*}{$\mathrm{p}$} \\
\hline & Good & Average & Poor & Good & Average & Poor & Good & Average & Poor & \\
\hline & $\begin{array}{c}\text { No. } \\
\%\end{array}$ & $\begin{array}{c}\text { No. } \\
\%\end{array}$ & $\begin{array}{c}\text { No. } \\
\%\end{array}$ & $\begin{array}{c}\text { No. } \\
\%\end{array}$ & $\begin{array}{c}\text { No. } \\
\%\end{array}$ & $\begin{array}{c}\text { No. } \\
\%\end{array}$ & $\begin{array}{c}\text { No. } \\
\%\end{array}$ & $\begin{array}{c}\text { No. } \\
\%\end{array}$ & $\begin{array}{c}\text { No. } \\
\%\end{array}$ & \\
\hline $\begin{array}{c}\text { Knowledge about chest } \\
\text { care procedures }\end{array}$ & $\begin{array}{c}0 \\
0.0\end{array}$ & $\begin{array}{c}0 \\
0.0\end{array}$ & $\begin{array}{c}20 \\
100\end{array}$ & $\begin{array}{c}12 \\
60.0\end{array}$ & $\begin{array}{c}8 \\
40.0\end{array}$ & $\begin{array}{c}0 \\
0.0\end{array}$ & $\begin{array}{c}0 \\
0.0\end{array}$ & $\begin{array}{c}3 \\
15.0\end{array}$ & $\begin{array}{c}17 \\
85.0\end{array}$ & $<0.001^{*}$ \\
\hline $\begin{array}{c}\text { Knowledge about } \\
\text { evaluation principles }\end{array}$ & $\begin{array}{c}0 \\
0.0\end{array}$ & $\begin{array}{c}0 \\
0.0\end{array}$ & $\begin{array}{c}20 \\
100 \\
\end{array}$ & $\begin{array}{c}14 \\
70.0\end{array}$ & $\begin{array}{c}6 \\
30.0 \\
\end{array}$ & $\begin{array}{c}0 \\
0.0\end{array}$ & $\begin{array}{c}3 \\
15.0\end{array}$ & $\begin{array}{c}11 \\
55.0\end{array}$ & $\begin{array}{c}6 \\
30.0 \\
\end{array}$ & $<0.001^{*}$ \\
\hline $\begin{array}{c}\text { Knowledge about } \\
\text { Self- evaluation } \\
\text { principles }\end{array}$ & $\begin{array}{c}0 \\
0.0\end{array}$ & $\begin{array}{c}0 \\
0.0\end{array}$ & $\begin{array}{c}20 \\
100\end{array}$ & $\begin{array}{c}0 \\
0.0\end{array}$ & $\begin{array}{c}0 \\
0.0\end{array}$ & $\begin{array}{c}20 \\
100\end{array}$ & $\begin{array}{c}0 \\
0.0\end{array}$ & $\begin{array}{c}1 \\
5.0\end{array}$ & $\begin{array}{c}19 \\
95.0\end{array}$ & $<0.001^{*}$ \\
\hline Total & $\begin{array}{c}0 \\
0.0\end{array}$ & $\begin{array}{c}0 \\
0.0\end{array}$ & $\begin{array}{c}20 \\
100\end{array}$ & $\begin{array}{c}0 \\
0.0\end{array}$ & $\begin{array}{c}11 \\
55.0\end{array}$ & $\begin{array}{c}9 \\
45.0\end{array}$ & $\begin{array}{c}3 \\
15.0\end{array}$ & $\begin{array}{c}2 \\
10.0\end{array}$ & $\begin{array}{c}18 \\
90.0\end{array}$ & $<0.001^{*}$ \\
\hline
\end{tabular}




\section{Tanta Scientific Nursing Journal}

Table (9): Levels of nurses total knowledge about chest care procedure, evaluation \& selfevaluation items pre, post and 3 month post program $(\mathrm{N}=80)$

\begin{tabular}{|c|c|c|c|c|c|c|c|c|c|c|}
\hline \multirow{3}{*}{ Knowledge items } & \multicolumn{3}{|c|}{ Pre program } & \multicolumn{3}{|c|}{$\begin{array}{c}\text { Immediate post } \\
\text { program }\end{array}$} & \multicolumn{3}{|c|}{ Post program } & \multirow{3}{*}{$\mathbf{p}$} \\
\hline & Good & Average & Poor & Good & Average & Poor & Good & Average & Poor & \\
\hline & $\begin{array}{l}\text { No. } \\
\%\end{array}$ & $\begin{array}{c}\text { No. } \\
\%\end{array}$ & $\begin{array}{c}\text { No. } \\
\%\end{array}$ & $\begin{array}{l}\text { No. } \\
\%\end{array}$ & $\begin{array}{c}\text { No. } \\
\%\end{array}$ & $\begin{array}{l}\text { No. } \\
\%\end{array}$ & $\begin{array}{c}\text { No. } \\
\%\end{array}$ & $\begin{array}{c}\text { No. } \\
\%\end{array}$ & $\begin{array}{l}\text { No. } \\
\%\end{array}$ & \\
\hline $\begin{array}{c}\text { Knowledge about } \\
\text { chest care } \\
\text { procedures }\end{array}$ & $\begin{array}{c}0 \\
0.0\end{array}$ & $\begin{array}{c}0 \\
0.0\end{array}$ & $\begin{array}{c}80 \\
100\end{array}$ & $\begin{array}{c}59 \\
73.8\end{array}$ & $\begin{array}{c}20 \\
25.0\end{array}$ & $\begin{array}{c}1 \\
1.3\end{array}$ & $\begin{array}{c}0 \\
0.0\end{array}$ & $\begin{array}{c}10 \\
12.5\end{array}$ & $\begin{array}{c}70 \\
87.5\end{array}$ & $<0.001^{*}$ \\
\hline \begin{tabular}{|c|}
$\begin{array}{c}\text { Knowledge about } \\
\text { evaluation } \\
\text { principles }\end{array}$ \\
\end{tabular} & $\begin{array}{c}0 \\
0.0\end{array}$ & $\begin{array}{c}2 \\
2.5\end{array}$ & $\begin{array}{c}78 \\
97.5\end{array}$ & $\begin{array}{c}57 \\
71.3\end{array}$ & $\begin{array}{c}19 \\
23.8\end{array}$ & $\begin{array}{c}4 \\
5.0\end{array}$ & $\begin{array}{c}7 \\
8.8\end{array}$ & $\begin{array}{c}45 \\
56.3\end{array}$ & $\begin{array}{c}28 \\
35.0\end{array}$ & $<0.001^{*}$ \\
\hline \begin{tabular}{|c}
$\begin{array}{c}\text { Knowledge self - } \\
\text { evaluation } \\
\text { principles }\end{array}$ \\
\end{tabular} & $\begin{array}{c}0 \\
0.0\end{array}$ & $\begin{array}{c}0 \\
0.0\end{array}$ & $\begin{array}{c}80 \\
100\end{array}$ & $\begin{array}{c}17 \\
21.3\end{array}$ & $\begin{array}{c}29 \\
36.3\end{array}$ & $\begin{array}{c}34 \\
42.5\end{array}$ & $\begin{array}{c}23 \\
28.8\end{array}$ & $\begin{array}{c}35 \\
43.8\end{array}$ & $\begin{array}{c}22 \\
27.5\end{array}$ & 0.368 \\
\hline Total & $\begin{array}{c}0 \\
0.0\end{array}$ & $\begin{array}{c}0 \\
0.0\end{array}$ & $\begin{array}{c}80 \\
100\end{array}$ & $\begin{array}{c}30 \\
37.5\end{array}$ & $\begin{array}{c}48 \\
60.0\end{array}$ & $\begin{array}{c}2 \\
2.5\end{array}$ & $\begin{array}{c}0 \\
0.0\end{array}$ & $\begin{array}{c}11 \\
55.0\end{array}$ & $\begin{array}{c}9 \\
45.0\end{array}$ & $<0.001^{*}$ \\
\hline
\end{tabular}

Table(10):Correlation between performing six chest nursing care procedures according to researcher's observation \& nurses' self-evaluation of their performance pre, immediate post \& 3 month post program $(\mathbf{N}=\mathbf{8 0})$

\begin{tabular}{|l|c|c|c|c|c|c||}
\hline \multirow{2}{*}{$\begin{array}{c}\text { Researcher observation } \\
\text { Chest nursing care procedures }\end{array}$} & \multicolumn{2}{|c|}{ Nurses self- evaluation performing chest nursing care procedures } \\
\cline { 2 - 7 } & $\mathbf{r}$ & $\mathbf{p}$ & $\mathbf{r}$ & $\mathbf{p}$ & $\mathbf{r}$ & $\mathbf{p}$ \\
\cline { 2 - 7 } & $0.772^{*}$ & $<0.001^{*}$ & $0.360^{*}$ & $0.001^{*}$ & $0.306^{*}$ & $0.006^{*}$ \\
\hline Turning procedure & $0.674^{*}$ & $<0.001^{*}$ & $0.593^{*}$ & $<0.001^{*}$ & $0.652^{*}$ & $<0.001^{*}$ \\
\hline Postural drainage procedure & $0.580^{*}$ & $<0.001^{*}$ & $0.552^{*}$ & $<0.001^{*}$ & $0.555^{*}$ & $<0.001^{*}$ \\
\hline Percussion procedure & $0.607^{*}$ & $<0.001^{*}$ & $0.498^{*}$ & $<0.001^{*}$ & $0.586^{*}$ & $<0.001^{*}$ \\
\hline Vibration procedure & $0.253^{*}$ & $0.024^{*}$ & $0.540^{*}$ & $<0.001^{*}$ & $0.542^{*}$ & $<0.001^{*}$ \\
\hline Coughing exercise & $0.650^{*}$ & $<0.001^{*}$ & $0.511^{*}$ & $<0.001^{*}$ & $0.377^{*}$ & $0.001^{*}$ \\
\hline Suctioning procedure & & & & \multicolumn{2}{c|}{ Post program } \\
\hline \hline
\end{tabular}

r: Pearson coefficient

Vol. 18 No. 1 May, 2020 


\section{Tanta Scientific Nursing Journal}

Table (11) Correlation between performing six chest nursing care procedures according head nurse's observation and nurses' self-evaluation performances pre, immediate post \& 3 month post program $(\mathrm{N}=20)$

\begin{tabular}{|c|c|c|c|c|c|c|}
\hline \multirow{3}{*}{$\begin{array}{l}\text { Head nurses observation } \\
\text { chest nursing care procedures }\end{array}$} & \multicolumn{6}{|c|}{$\begin{array}{l}\text { Nurses self- evaluation } \\
\text { Frequency of performing chest nursing care procedures }\end{array}$} \\
\hline & \multicolumn{2}{|c|}{ Pre program } & \multicolumn{2}{|c|}{$\begin{array}{l}\text { Immediate post } \\
\text { program }\end{array}$} & \multicolumn{2}{|c|}{ Post program } \\
\hline & $\mathbf{r}$ & $\mathbf{p}$ & $\mathbf{r}$ & $\mathbf{p}$ & $\mathbf{r}$ & $\mathbf{p}$ \\
\hline Turning procedure & $0.538^{*}$ & $0.015^{*}$ & 0.559 & 0.010 & 0.409 & 0.074 \\
\hline Postural drainage procedure & $0.745^{*}$ & $<0.001^{*}$ & 0.029 & 0.905 & 0.013 & 0.957 \\
\hline Percussion procedure & $0.801^{*}$ & $<0.001^{*}$ & 0.402 & 0.079 & $0.596^{*}$ & $0.006^{*}$ \\
\hline Vibration procedure & 0.345 & 0.137 & 0.222 & 0.346 & 0.312 & 0.180 \\
\hline Coughing exercise & 0.355 & 0.124 & 0.479 & 0.033 & 0.323 & 0.165 \\
\hline Suctioning procedure & $0.567^{*}$ & $0.009^{*}$ & 0.335 & 0.148 & $0.457^{*}$ & $0.043^{*}$ \\
\hline
\end{tabular}

r: Pearson coefficient *: Statistically significant at $p \leq 0.05$ 


\section{Tanta Scientific Nursing Journal}

\section{Discussion}

Head nurses evaluation of nurses performance of chest care procedures guarantee high quality care to achieve desired patient outcomes as chest care is an integral aspect of the nursing care of patient suffering with respiratory conditions .Procedures of chest care include turning, postural drainage, percussion, vibrations, coughing exercise and suctioning. Self-evaluation of chest care procedure performance give nurses deeper recognition about weakness points and required training. The aim of present research is to study nursing staff performance of the six chest care procedures and implement intervention program to expand nursing staff knowledge about standard steps for performing chest care procedures and, principles of evaluation and selfevaluation to raise their attention for improvement.

Result of preprogram revealed that minority of nursing staff showed excellent level of total performance of six chest care procedures. Most probably their diploma education level and not attending training program affect negatively their performance level. Actually they have poor knowledge about standard steps of chest care procedures performance, protocols, and technique used, especially for turning and chest percussion. Apparently those nursing staff are in need for improving and updating their knowledge through inservice educational program emphasize the importance of following standard of care and evidence-based practices in performing chest care procedures specially emphasize importance of head nurses supervision to evaluate nurses' performance and repair their weakness to meet quality chest patient care .

Head nurses supervision is considered to have three principal functions including; educational, supportive and managerial functions that could help in attaining personnel and hospital goals ( ${ }^{97)}$.It is an important way to support and guide nurses to ensure safe practice and quality of care and helping them in coping with their working situations. Really it helps nurses to maintain their ability to take action under stress and to adopt a more tolerant attitude towards patients. Head nurses supervision has an effect on the quality of care by helping to both improve and maintain of professional standards of nursing care and the nursing practice can be improved through their education of Fawzi (2015) ${ }^{(18)}$ conducted a study to explore factors affecting application of infection control measures supported 


\section{Tanta Scientific Nursing Journal}

present study and agree with Osman $\mathbf{( 2 0 1 4 )}^{(\mathbf{1 9})}$ study about " assessment of nurses performance caring for patient. Both revealed that the majority of nurses had unsatisfactory knowledge and practice regarding infection control measures due to that all of studied nurses didn't receive training courses about chest infection due to lack of in-services training programs and lack of awareness about training courses that improve their performance. care and supervision.

A smaa ( 2017) ${ }^{(\mathbf{2 0})}$ conducted a study about nurses performance regarding management of patient with chest infection in neuro -critical care unit in relation to level of nurses knowledge and practices and their demographic characteristic illustrated statistically significant relation between education level,years of experience and total knowledge level .

Mahran etal (2018) (21) comparative study of critical nurses' knowledge and practice before and after education program about acute exacerbation of chronic obstructive pulmonary disease. Support present study and pointed that the improvement of nurses knowledge was reflected on their performance which was assessed after the education program. Significant improvement was found in the performance of nurses and application of chest care procedure standard after program implementation

Main, Denehy ( 2016$)^{(22)}$, study about physiotherapy for respiratory and cardiac problems - adults and pediatrics, supported the present study and reported that implementation of standard, with proper understanding of postural drainage basic steps and principle through proper knowledge, revealed to be essential to make it simple and more effective and lead to desirable patient out come . Also Ciesla, (2011) ${ }^{(23)}$ conducted a study on the effectiveness of frequent practice of postural drainage on the clients with respiratory disorders pneumonia, revealed that nurses performance of postural drainage helped in the beneficial effects for removal of retained secretions and improved depending on nurses performance and degree of their knowledge .

Emelia .etal (2018) ${ }^{(24)}$ study about intensive care nurses' knowledge and practice on endotracheal suctioning of the intubated patient, and Fisk. (2018) ${ }^{(25)}$ study about the effects of endotracheal suctioning in pediatric population .Both studies showed that majority of ICU nurses knew the indication for procedure but, most of nurses demonstrated undesirable overall knowledge on endotracheal 


\section{Tanta Scientific Nursing Journal}

suctioning (ETS) and no one of nurses was on the desired performance level .Nurses with educational training program significantly demonstrated higher knowledge of ETS than non-trained nurses Dehghani (2014) ${ }^{(26)}$ the study about investigating intensive care units nurses performance and its adjusting with standard, results of a study on suctioning revealed nurses' poor knowledge and performance and non-compliance of suctioning procedure with the standard method. Also Varghese and, Moly . (2016) ( 27) exploratory study on the knowledge and skill of critical care nurses on endotracheal suctioning, showed deficit areas of knowledge and skill in specific phases of suctioning as well as a significant difference between the current practices observed and best recommended practice on suctioning.

Bhat et al., (2014) ${ }^{(28)}$ study about chest physiotherapy techniques in neurological intensive care units of India: reported that the availability of resources is one of the important factors that influence chest care practice in the ICU in terms of equipment availability, training of the nurses , awareness of techniques and standard and , nurses to patient ratio at ICUs Also Leligdowicz, (2016) (29), Study about development of an ICU resource assessment survey for the care of critically ill patients in resource-limited settings, reported that an intensive care unit is an isolated confined ward in the hospital where the most critically ill patients are located together and managed using specialized trained nurses able to use chest care procedures equipment for helping patient

Fedorovich and, Littleton .(2017) $)^{(30)}$, study about chest physiotherapy: evaluating the effectiveness. Dimensions of critical care nursing, that study has particularly shown the effects on chest care procedures evaluation, and assured that specifically trained respiratory nurses are key to ensuring quality care. A respiratory nurse may be staff nurse, nurse practitioner and supervisor .Adding that an increasingly important element of the specialist respiratory nurse's role is to provide patients with information at patients' level of understanding, and are trained in patient communication skills, and the techniques relevant to respiratory chest care procedures to gain satisfactory nurses performances and good level of patient outcome.

While Farida (2017) ${ }^{(31)}$ Study to asses factors affecting nurses performance in selected governmental hospitals in Egypt, concluded that the most factors affecting 
on nurses performance was head nurses supervision and evaluation of their performance. Abd - El-Halem, (2013) ${ }^{(32)}$, Fulton et al., (2014) $)^{(33)}$, and Morsi, (2014) $^{(34)}$ from their studies found a highly statistically significant improvement in nurses' knowledge immediately after program implementation, training had a positive effect in improvement of head nurses knowledge as the knowledge they gained improve their supervision competencies. Hossein Abadi, etal (2015) (35) study about effect of multi mentoring educational method on clinical competence of nursing. The results obtained show that constant evaluation of nurses' competency is one of the most principal responsibilities of head nurses.

\section{Mohsen and Azade (2013) ${ }^{(36)}$ Study} about nurses self -evaluation of their use and mastery in health assessment skills. Assessment of clinical skills is usually performed by head nurses. However, nurses self-evaluation of their caring skills is stressed in recent years. It is believed that monitoring the professionals' behavior with self-assessment help them develop skills, assist them becoming more independent and confident and empower them to select higher goals and to try to realize these goals and finally assist them to improve and strengthen their skills.
The importance of self- evaluation in professional life and also developed an indepth analysis on how self-performance can be developed. Many practical strategies were highlighted to develop selfevaluation at individual and professional level. In conclusion, self- evaluation is considered as the therapeutic tool for nurse client relationship. The more the nurse will be self-aware of performance the more a therapeutic environment for caring will be enhanced. Therefore, development of selfevaluation, knowing about oneself performance is not an easy task; it is a painful and time consuming process.

This process starts with conscious awareness of performance and struggling for change through continuous efforts. There is no doubt that Self- evaluation is one of the important components in nurse client relationship. Nurses spend most time with the patients than of any other health care professionals so self-evaluation is considered as an important tool to develop a therapeutic relationship with the client. Examine the aspects of self- performance and get in depth understanding of this concept. In addition explore the practical implications of self- awareness of performance in nursing profession.

Present study correlation data revealed significant positive correlation in 


\section{Tanta Scientific Nursing Journal}

performing six chest nursing care procedure according to researcher observation, head nurses evaluation and nurses self -evaluation pre, post and 3 month post. This at pre- program related to head nurses and nurses insufficient knowledge about standard, performance of six chest care procedures and ,evaluation and self- evaluation principles .But for post program data apparently ,because they understand steps of six chest care procedures performance, they know each of chest care procedure assessment , indication, contraindication, preparation of equipment, preparation, communication and teaching for patient, implementation of each procedure with following infection control and evaluation of their desirable and desirable outcome .

Specially head nurses start to make evaluation for nurses performance using plan, information, principles and methods of making evaluation .Regarding nurses they gradually improved for performing six chest care procedures following standard and self- evaluating their performance. So implementation of the evaluation program was successful for improving nursing staff knowledge and performance of six chest care procedures. Also the program assisted head nurses to make skillful evaluation for nurses' performance of six chest care procedures .As well as assisted nurses for making self- evaluation skillfully for their performance of chesty care procedures. Really the program was effective to improve knowledge and performance of nursing staff about steps of performing chest care procedure, evaluation and self evaluation of that performance.

\section{Conclusion}

Head nurses and nurses at Tanta university hospitals in Mobark, chest and international educational hospital were lacking knowledge about principles of chest care six procedures, evaluation of nurses performance of chest care six procedures was at unsatisfactory level and they not follow the standard steps for performing any of chest care procedures Beside head nurses were not making evaluation for nurses performances ,as well as nurses were not making self- evaluation for their performance regarding chest care procedures .Implementation of successful educational program and standard of performing chest care procedure. The program knowledge enforced head nurses to recurrently evaluate nurses performance and evoked nurses for periodically evaluate their performance, correct their faults and strengthen their weakness points. Consequently nurses performance of six chest care procedures improved to be 
satisfactory level post program due to nurses following standard steps of performance, periodic self -evaluation and head nurses evaluation of nurses performance. Suggested recommendation Based on the present study finding, the following recommendation are proposed :

1- Conduct regular training programs workshops and seminars for head nurses to updated their knowledge and skills related to nurses supervision and evaluation of their performance.

2- Conduct periodical in service training program for nurses to refresh their knowledge and skills related to chest care procedure performance according to standard of its performance.

3- Stress the use of regular self -evaluation among nurses at intensive care units to correct their defect in performance of chest care procedures.

4- Conduct orientation programs about standard of chest care procedures for novice nurses before working with chest disease patients.

5- Hospital provide adequate resources and equipment to facilitate nurses performance according to standard of performing chest care procedures.

6- Head nurse stress importance of good communication and teaching for patient among nurses at intensive care units.
7- Head nurses provide adequate regular and timely feedback to nurses concerning their weakness points and correct their wrong or deficient performance.

8- Head nurses should encourage nurses' compliance for infection control measure in performing chest care procedures.

9- Head nurses should provide supportive health work environment to help nurses finding positive new ways to improve quality of chest care procedures performance.

10- The hospital should set clear roles and responsibilities for both head nurses and nurses through a constructive supervision policies, feedback, and support procedures standard implementation.

11- The hospital should encourage a close rapport between head nurses and their nurses for improving their implementation of both evaluation and self evaluation

Recommend research

- Assess barriers for nurses' self-evaluation of performing chest care procedure

- Evaluation and self -evaluation must put in spot light for different studying aspects. 
- Replication of current study in other hospitals setting

- Study attributes that promote the development of self-evaluation among nurses.

\section{References}

1- Ogata Y. Examination of Performance Reflection in Nursing Practice: A focus on Improvement of Nursing Judgement. Hokkaido Iryo Daigaku Kango Fukushi Gakubu Gakkaishi. 2014;10:43-7. Japanese.[Google Scholar]

2- Arabi, Y. M., Phua, J., Koh, Y., Du, B., Faruq, M. O., and Nishimura M. Structure, organization, and delivery of critical care in Asian ICUs. Critical Care Medicine. 2016; 44(10): 940- 948.

3- Moghaddam N.M., Jame SZB., Rafiei S., Sarem AA., Ghamchili A., and Shafii M. Managerial competencies of head nurses: a model and assessment tool. Br J Nurs. 2019 Jan 10;28(1):3037. doi: 10.12968/bjon.2019.28.1.30.

4- Hani M., and Ahmad M. Evaluation of the concept of holism in nursing care. Persian Journal of Medical Sciences. 2016;3:4. [Google Scholar]

5- Bruce Martin, Jeffrey J. McNally and Simon Taggar: Determining the importance of self-evaluation on the goal-performance effect in goal setting:
Primary Findings

Article (PDF

Available) in Canadian Journal of

Behavioural Science 48(2) · January 2015; with 685

DOI:

$10.1037 / \mathrm{cbs} 0000025$

6- Kudo M., Komatsu M., Omi S., Nakayama Y., Ohira M., and Mashita A.Nursing management's evaluation and expectation of competencies in nurses with long-term clinical practice experiences.Proceedings of the 36th Japan Academy of Nursing Science Academic Conferences. 2016;499. Japanese.[Google Scholar]

7- Hamdan M. Albaqawi, Vincent R. Butcon, and Roger R. Molina. Awareness of holistic care practices by intensive care nurses in north-western Saudi ArabiaSaudi Med J. 2017 Aug; 38(8): 826-831. doi: 10.15537/smj.2017.8.20056

PMCID: PMC5556299 PMID: 28762435

8- Elizabeth H Skinner, Kimberley J Haines PT, Sue Berney, Stephen Warrillow, Meg Harrold, and Linda Denehy . Usual Care Physiotherapy During Acute Hospitalization in Subjects Admitted to the ICU: An Observational Cohort Study. 2015; Australia. E-mail: elizabeth.skinner@ wh.org.au. DOI: 10.4187/respcare 
.040641476 RESPIRATORY CARE • OCTOBER 2015 VOL 60 NO 10

9- Jones T., "Outcome Measurement in Nursing: Imperatives, Ideals, History, and Challenges". OJIN: The Online Journal of Issues in Nursing .May 31, 2016; 21(2): Manuscript 1.DOI: 10.3912/OJIN.Vol21No02Man01

10- Genuino M., J. Effects of simulation-based educational program in improving the nurses' self-efficacy in caring for patients' with COPD and $\mathrm{CHF}$ in a post-acute care (PACU) setting. Applied Nursing Research. $2018 ;(39): 53-57$.

11- Rehab Hani Elkazaz, Abeer Elsayed Berma. Stress and self-efficacy among nurses in neonatal intensive care unit, Port Said University, Egypt. 2017;5(2) DOI:

https://doi.org/10.5281/zenodo.345678 [Elkazaz et. al., Vol.5 (Iss.2): February, 2017] ISSN- 2350-0530(O), ISSN- 2394-3629(P) ICV (Index Copernicus Value) 2015: 71.21 IF: 4.321 (CosmosImpactFactor), 2.532 (I2OR) InfoBaseIndex IBI Factor 3.86

12- Kimura M., Nishikawa M., Akutagawa K., Kataoka M.,and Hayashi M. Bibliographic consideration of educational methods and evaluation for the development of nursing competency. Hiroshima Kokusai Daigaku Kangogaku Journal. 2012;9:25-34. Japanese with English abstract. [Google Scholar]

13- VanFosson C. A., Jones T. L., and Yoder, L. H. Unfinished nursing care: An important performance measure for nursing care systems. Nursing Outlook, 2015; 64(2): 124-136. doi:10.1016/joutlook.2015.12010

14- Langenderfer B. Alternatives to Percussion and Postural Drainage. A Review of Mucus Clearance Therapies: Percussion and Postural Drainage, Autogenic Drainage, Positive Expiratory Pressure, Flutter Valve, Intrapulmonary Percussive Ventilation, and High-Frequency Chest Compression with The Therapy Vest. J. Cardiopulmonary Rehabilitation. 1998; 18:283-289.

15- Gass E, "Patterns of Chest Physiotherapy in Australian Intensive Care Units. Journal of Critical Care. (2009); 19(3):145-151.

16- Jose Antoni Lammogila Microsoft Clinical Handbook Respiratory Therapy Program. UTMB Respiratory Care Services (Procedure Chest Physiotherapy) page 1 of 10 Policy 7.3.9.(2005).Jlammoglia@mm.fnc. edu .pcms?pid . 
17- Walsh M. and Crumbie. What Sons Clinical Nursing and Related Sciences Caring for The Patient with Respiratory Disorder .Edin burg London New Yourk Oxford ,Philadelphia, St Louis Sydney Toronto, $7^{\text {th }}$ ed. 2007; ppt325-13.

18- Ramazini M Fawzi . Factors Affecting Application of Infection Control Measures.. Professional Nursing Today.2015;24(3):6-2 .IntJ.Pediatic https://hdl.handle.net/ 10520/EJC79308

19- Osman IH. , Berbar LN., and Sidani Y. Data envelopment analysis model for the appraisal and relative performance evaluation of nurses at an intensive care unit. Journal of Medical Systems. 2011; 35(5): 1039-1062. https://doi.org/10.1007/s10916-010-9 570-4

20- A smaa - Nurses performance regarding management of patient with chest infection in neuro -critical care unit International Journal of Nursing Science 2017; 5(2): 66-75.

21- Main E, Denehy L. Physiotherapy for respiratory and cardiac problems Adults and paediatrics. Fifth. Elsevier, 2016. [Google Scholar]

22- Ciesla PT. Effectiveness of frequent practice of postural drainage on the clients with respiratory disorders pneumonia 2011;16 (35):1-82. [PubMed] [Google Scholar] 212011595 (USA) (nciesla@erols.com).

23- Emelia T. Mwakanyanga, Golden M. Masika, and Edith A. M. Intensive care nurses' knowledge and practice on endotracheal suctioning of the intubated patient: A quantitative crosssectional observational study. Published: August 16, 2018 .https://doi.org/10.1371/journal.pone.0 201743

24- FiskAC. The effects of endotracheal suctioning in the pediatric population: An integrative review. Dimens Crit Care Nurs. 2018;37(1):44-56. pmid:29194174

25- Dehghani K., Nasiriani K., and Mousavi T. Investigating intensive care unit nurses' performance and its adjusting with standard. SSU Journals. 2014;21:808-15. [Google Scholar]

26- Varghese ST., and Moly KT . Exploratory study on the knowledge and skill of critical care nurses on endotracheal suctioning. The Journal of National Accreditation Board for Hospitals \& Healthcare Providers, 2016, 3(1): 13. 
27- Sigera P. C., Tunpattu, M. U. S., Jayashantha, T. P., De Silva, A. P., Athapattu, P. L., Dondorp, A., and Haniffa, R. (2016). National profile of physiotherapists in critical care units of Sri Lanka: A Low-Middle Income Country. Physical therapy. doi:102522/ptj.20150363.National profile of nurses physiotherapies in critical care unit Sir lanka.

28- Bhat, A., Chakravarthy K., and Rao, B. K. Chest physiotherapy techniques in neurological intensive care units of India: A survey. Indian Journal of Critical Care Medicine: 2014; 18(6): 363-368.

29- Leligdowicz, A., Bhagwanjee, S., Diaz, J. V., Xiong, W., Marshall, J. C., Fowler, R. A., and Adhikari, N. K. Development of an ICU resource assessment survey for the care of critically ill patients in resourcelimited settings. Journal of Critical Care. 2016 ; 38, 172-176.

30- Fedorovich C., Littleton MT.Chest physiotherapy: evaluating the effe124Farida .Study to asses factors affecting nurses performance in selected governmental hospitals in Egypt . Infect Control Hosp Epidemiol. 2017; 27(6) : $571 \quad-5$ [DOI][PubMed]

31- Abd El-Halem, E., H. (2013): Educational program for nurses about pregnancy induced hypertension in Port Said, Doctorate thesis, University of Port Said, Faculty of Nursing,
Egypt. Available at: www.iosrjournals .org.

32- Fulton et al. Factors affecting performance of professional nurses in north west bank governmental hospitals. J Educ Pract 2014; 6(7): 100-10.

http://iiste.org/Journals/index.php/JEP.

33- Farida .Study to asses factors affecting nurses performance in selected governmental hospitals in Egypt - Infect Control Hosp Epidemiol. 2017; 27(6) : $571 \quad-5$ [DOI][PubMed]

34- Morsi, M.The Effectiveness of implementing clinical supervision models on head nurses' performance and nurses' job satisfaction at Benha University Hospital. Faculty of Nursing, Benha University. (2014).

35- 34-Hossein Abadi R., Gholami M., Biranvand Sh., Tarvedian A., and Anbari Kh. Effect of multi mentoring educational method on clinical competence of nursing students. J Med Edu Dev 2015; 10(2): 119-128. [Persian] ctiveness. Dimensions of Critical Care Nursing, Mar-Apr 2017; 9(2): 68-74.

36- Mohsen Adib-Hajbaghery, and Azade Safa "Nurses' evaluation of their use and mastery in health assessment skills: selected Iran's Hospitals. Nurs Midwifery Stud. 2013 Sep; 2(3): 3943. PMCID: PMC4228544 PMID : 25414875 